\title{
Experimental and analytical study of dual compensation chamber loop heat pipe under acceleration force assisted condition
} \author{
Longzhu Han a , Yongqi Xie ${ }^{\text {b,* }}$, Jianqin Zhuc ${ }^{\mathrm{c}}$, Hongwei $\mathrm{Wu}^{\mathrm{d}, * *}$, Hongxing Zhang ${ }^{\mathrm{e}}$ \\ ${ }^{\text {aS }}$ chool of Biological Science and Medical Engineering, Beihang University, Beijing, 100191, China \\ ${ }^{\mathrm{b}}$ School of Aeronautic Science and Engineering, Beihang University, Beijing, 100191, China \\ ${ }^{c}$ National Key Laboratory of Science and Technology on Aero-Engines, School of Energy and Power Engineering, \\ Beihang University, Beijing 100191, China \\ ${ }^{\mathrm{d}}$ School of Engineering and Computer Science, University of Hertfordshire, Hatfield, AL10 9AB, United Kingdom

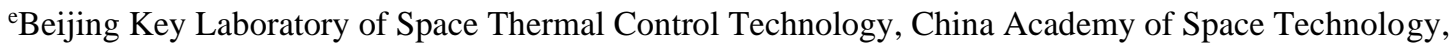 \\ Beijing 100094, China \\ *Corresponding author. Yongqi Xie, Email: xyq@buaa.edu.cn ${ }^{* *}$ Corresponding author. Hongwei Wu, Email: h.wu6@herts.ac.uk
} Abstract: In this article, a combined experimental and theoretical study has been conducted to investigate the operating characteristics of a dual compensation chamber loop heat pipe (DCCLHP) with ammonia as the working fluid under acceleration force conditions. The DCCLHP with nickel wick consists of a cylindrical evaporator and dual compensation chambers at both ends of evaporator. In the current study, a new DCCLHP test rig was setup which can provide the acceleration up to $11 \mathrm{~g}$. Two types of loading mode were utilized for applying heat load prior to acceleration. The heat load ranges from $25 \mathrm{~W}$ to $300 \mathrm{~W}$. Comparisons of operating performance of the DCCLHP were carried out under both gravity and acceleration conditions. A novel centrifugal force assist concept is proposed to address the observed operating behavior. Experimental results show that: (i) the acceleration effect with the proposed orientation could improve the operation performance of the DCCLHP which may operates at the centrifugal force driven mode and capillary-centrifugal force co-driven mode. The operating temperature profile at different heat loads shows "/-shape" oblique line with the increase of the acceleration; (ii) the transition heat load from centrifugal force driven mode to capillary-centrifugal force co-driven mode changes with the variation of acceleration magnitude at both loading modes; (iii) the acceleration effect on the operating temperature is remarkably significant as the heat load is less than $100 \mathrm{~W}$. The operating temperature under acceleration conditions is apparently lower than that under terrestrial gravity; (iv) the coupling change of the loop pressure, vapor-liquid distribution, two-phase flow and heat transfer caused by acceleration effect leads to the unique operation performance of the DCCLHP.

Keywords: loop heat pipe; dual compensation chamber; operating characteristics; elevated accelerated force; electronic cooling. 
$35 a \quad$ Radial acceleration $\left(\mathrm{m} / \mathrm{s}^{2}\right)$

$36 g$ Gravitational acceleration $\left(9.81 \mathrm{~m} / \mathrm{s}^{2}\right)$

$37 \quad G \quad$ Thermal conductance (W/k)

$38 h \quad$ Latent heat of vaporization $(\mathrm{J} / \mathrm{kg})$

$39 \quad H \quad$ Length $(\mathrm{m})$

$40 \quad \Delta H \quad$ Distance $(\mathrm{m})$

$41 m$ mass flow rate $(\mathrm{kg} / \mathrm{s})$

$42 \quad Q \quad$ Heat load (W)

$43 \quad R \quad$ Pore radius (m)

$44 \quad T \quad$ Temperature (K)

$45 \Delta T \quad$ Temperature difference $(\mathrm{K})$

$46 \Delta p \quad$ Pressure difference $(\mathrm{Pa})$

47 Greek symbols

$48 \quad v \quad$ Specific volumes $\left(\mathrm{m}^{3} / \mathrm{kg}\right)$

$49 \theta \quad$ Contact angle (arc degree)

$50 \rho \quad$ Density $\left(\mathrm{kg} / \mathrm{m}^{3}\right)$

$51 \sigma \quad$ Surface tension $\left(\mathrm{N} / \mathrm{m}^{2}\right)$

52 Subscripts

53 a $\quad$ Acceleration

54 bay Bayonet tube

55 cond Condenser

56 e Evaporator

$57 \quad 11 \quad$ Liquid line

$58 \quad v g \quad$ Vapor and liquid

$59 \mathrm{vl} \quad$ Vapor line

60 in At inlet

61 cap, max Maximum capillary pressure

62 out At outlet

$63 \mathrm{r} \quad$ Radial 
Saturation

total

Total

66

$\mathrm{W}$

Wick

67 Acronyms

68

CC Compensation chamber

69

CCM Constant conductance mode

DCCLHP Dual compensation chamber loop heat pipe

LHP

Loop heat pipe

RTD

Resistance temperature detector

$73 \quad \mathrm{VCM}$

Variable conductance mode

\section{Introduction}

As a passive closed two-phase heat transfer device, loop heat pipes (LHPs) exhibit extremely attractive performance in many energy-related applications with the features of self-starting, flexibility, high efficiency and long distance heat transport [1-3]. They use the capillary pressure to drive the circulation flow and the heat transfer is transported from one location to another. Since the widely successful applications in the aerospace industry [4-6], the focus and interest of LHPs have been shifted towards the terrestrial and aeronautics applications [7-9].

With more and more terrestrial applications in heat transfer areas, the effect of the gravitational force on the LHP performance has become much more important. Over the past two decades, many research efforts have been devoted to the LHP behaviors under positive or adverse elevation, which refers to the condenser above or below the evaporator [10-13]. Chen et al. [14] experimentally studied the performance of a miniature LHP with a cylindrical evaporator for horizontal and four vertical orientations. They presented that the steady-state operating performance was similar for different orientations with $132 \mathrm{~mm}$ positive and adverse elevation. There was a high start-up temperature under positive elevation. However, the start-up failed under adverse elevation at the sink temperature of $15{ }^{\circ} \mathrm{C}$. At low heat load, the LHP operating temperature increased with the adverse elevation. The reason has been explained by $\mathrm{Ku}$ [15]. Due to gravitational head effect, the pressure difference across the wick increased, so would the saturation temperature difference. It successively resulted in an increased heat leak. Since the 
amount of subcooling of the liquid returning back to the compensation chamber (CC) did not change, the $\mathrm{CC}$ temperature increased to provide enough subcooling to balance the increased heat leak. Chuang $[16,17]$ firstly proposed the operating theory under gravity-assisted conditions on the basis of the experimental data and visualization observations. The operating temperature profiles at $25.4 \mathrm{~mm}, 76.2 \mathrm{~mm}$ and $127.0 \mathrm{~mm}$ positive elevation were explained in detail in the pressure-temperature diagram. Flow visualization results successfully validated the theory and clearly showed vapor-liquid two-phase flow in the vapor line as operating at gravity-controlled mode. When the total pressure drop of the system was lower than the maximum gravitational pressure head, this pressure head itself was enough to circulate the flow in the loop. The pores of the wick were filled with liquid and there was no meniscus. Riehl [18] performed a series of tests on the acetone LHP under horizontal position and with the evaporator above or below the condenser. It was found that the LHP could reliably operate at all situations even at the heat load as low as $1.0 \mathrm{~W}$. Comparing to the horizontal position, there were higher operating temperatures under the evaporator above and lower operating temperatures under the evaporator below. Chang et al. [19] carried out visualization study of a LHP with two evaporators and one condenser under gravity-assisted condition. They found that the heat leak ratio and two-phase flow region at gravity-driven mode were smaller and longer than those at capillary-gravity codriven mode except at 10/10 W when the condenser was filled with liquid. The heat load sharing phenomenon for two evaporators disappeared at the gravity-driven mode because of the effect of the gravitational head.

A comprehensive 1-D steady-state model of an ammonia LHP was developed by Chuang et al. [20] to predict the operating temperature under both zero and adverse elevations. The comparisons with experimental data showed in good agreement. But under the positive elevations, large deviation from the test data was observed. The gravity effects on the operating performance of the LHP with flat evaporator have been investigated experimentally by Mo et al. [21] for four different orientations. It was found that a higher operating temperature and thermal resistance occurred at adverse elevation but a lower operating temperature and thermal resistance at positive elevation. Moreover, the temperature oscillations were observed at positive elevation. Bai et al. [22] experimentally studied the steady-state operating characteristics of an ammonia-stainless LHP at gravity-assisted attitude and compared with the results under horizontal and adverse 
elevation attitudes. They proposed two driving modes as operating under gravity-assisted attitude: gravity-driven mode and capillary-gravity co-driven mode. According to the system pressure balance and the energy balance inside the $\mathrm{CC}$, they explained the reasons of the lower operating temperature under the gravity driven mode in detail. Afterwards, they [23] further established a steady-state mathematical model of a LHP under gravity-assisted operation in terms of both driving modes and validated against the experimental data. The variations of transition heat load and mass flow rate, steady-state operating temperature and thermal conductance under various positive elevations were analyzed. They also found that the thermal conductance of the LHP increased with the increase of positive elevation, especially in the variable conductance region. In their latest study [24], they confirmed that dual compensation chamber LHP (DCCLHP) with an extended bayonet tube could enhance the cooling to CCs and successfully start up at different heat loads in the horizontal orientation. For an ammonia-stainless steel DCCLHP, Feng et al. [25] experimentally investigated the operating instability under different orientations. The temperature hysteresis and temperature oscillations were observed under gravity-assisted elevations and antigravity elevations. The thermal vacuum test of the LHP with the condenser above the evaporator and CC was conducted and the operating behavior was studied by Ku et al [26]. It was found that the requirement of control heater power on the $\mathrm{CC}$ was much higher than that predicted. The essential cause was the fluid flow and CC temperature oscillations, which was caused by the interaction between gravity and $\mathrm{CC}$ heating, and was deteriorated by the variable gravitational pressure head.

Different from the conditions in the gravity field, the LHP operating in the acceleration fields can show some unique operating characteristics. It is recognized that experimental studies on the effect of acceleration on the operating characteristics are very limited. Utilizing a spin table to examine the various acceleration effects on start-up performance, Ku et al. [27] carried out several different experiments on a miniature anhydrous ammonia-aluminum LHP under two mounting configurations. One was horizontal with the $\mathrm{CC}$ and liquid line outboard on the spin table, the other was horizontal with the evaporator and vapor line outboard on the spin table. The conditions included LHP start-up before applying acceleration and vice versa. The acceleration profiles consisted of constant radial acceleration $1.2 \mathrm{~g}$ and $4.8 \mathrm{~g}$, as well as the combination of these both acceleration magnitudes. Their results revealed that the LHP could start up successfully in all 
experiments. The wall superheat was independent of input heat load and acceleration. As an extension of the previous investigation, they [28] also studied the temperature stability of the same LHP under different acceleration and heat load conditions. It was observed that the acceleration force led to the redistribution of the working fluid in the evaporator, condenser and $\mathrm{CC}$, which affected the operating performance finally. In each experiment, the LHP could operate normally.

According to the investigations on the operating performance of a titanium-water LHP under standard and acceleration fields conducted by Fleming et al. [29], it was found that dry-out conditions occurred at varying radial accelerations from $0 \mathrm{~g}$ to $10 \mathrm{~g}$ for the heat loads from 100 $\mathrm{W}$ to $400 \mathrm{~W}$, but did not occur at $400 \mathrm{~W}$ and $600 \mathrm{~W}$ under $10 \mathrm{~g}$ conditions. Periodic fluid flow reversal was observed for some cases. The evaporative heat transfer coefficient and thermal resistance were slightly dependent on the radial acceleration. It should be noted that the acceleration vector directed from the evaporator to the condenser. Yerkes et al. [30] used a titanium-water LHP with the same design parameters as studied by Fleming et al. [29] to investigate the steady periodic sine acceleration effects on the operating performance. The radial acceleration magnitude and frequency ranged from $0.5 \mathrm{~g}$ to $10 \mathrm{~g}$ and $0.01 \mathrm{~Hz}$ to $0.1 \mathrm{~Hz}$, respectively. The heat load was from $300 \mathrm{~W}$ to $600 \mathrm{~W}$. Their results revealed that the acceleration force complemented the thermodynamic force to improve the LHP dynamic performance but the converse was always true as the acceleration force countered the thermodynamic force in some cases. In their further study on the transient operating behavior of a titanium-water LHP [31], a phase-coupled evaporator heat load to acceleration were produced as periodic sine functions with a fixed frequency. The dynamic performance at different condenser temperatures were evaluated at various heat loads and acceleration loads. It was believed that the nature frequency of the fluid motion inside the condenser could cause the delayed failure of the LHP. In our previous work [32, 33], the steady-state and transient operating performances of a DCCLHP at four different horizontal arrangements have been studied as the heat load and acceleration force were applied simultaneously and the heat load was applied firstly until the loop reached a steady state and then the acceleration force was applied. It was found that the operating behaviors under the arrangement of the evaporator relative to the condenser locating at the outboard of the rotational arm were evidently distinct from those under the other three arrangements. The effect at the 
special arrangement was similar with that at gravity-assisted position. The centrifugal force became the driving force to pump the circulation flow.

To the best of the authors' knowledge, there are no open published reports on the theoretical and/or experimental studies on the operating characteristics of the DCCLHP under acceleration fields where the acceleration effect contributed to the returning liquid going back to the CC. Therefore, in the present work, the operating characteristics of the DCCLHP under the acceleration assisted conditions were further investigated experimentally. Two types of loading mode were used for the heat load and acceleration load, i.e., applying the heat load until the loop getting to a steady state and then applying the acceleration and simultaneously applying the heat load and acceleration. In the current study, a novel acceleration force assisted concept is proposed to explain the observed unique phenomena, in which the loop may operate at the centrifugal force driven mode and centrifugal-capillary co-driven mode. The influences of different heat loads and acceleration magnitudes on the operating behavior were analyzed in a systematical manner. The effect of the acceleration magnitudes on the transition heat load separating the DCCLHP operation into centrifugal force driven mode and capillary-centrifugal force co-driven mode at both loading modes was discussed. The results would be helpful for deep understanding the LHP operating mechanism under acceleration fields.

\section{Experimental setup}

In the current work, an ammonia-stainless strain DCCLHP is designed and manufactured. An acceleration simulation test rig is established at the Reliability and Environmental Engineering Laboratory at Beihang University, Beijing, China. The test rig can be utilized to experimentally investigate the acceleration force-assisted operating behavior of the DCCLHP under acceleration fields.

\subsection{Test section}

Fig. 1 presents a photo of the ammonia-stainless steel DCCLHP and the internal construction of the evaporator and CCs, which is manufactured by China Academy of Space Technology. The overall dimension of the DCCLHP is $565 \mathrm{~mm}$ (Length) $\times 469 \mathrm{~mm}$ (Width) $\times 27 \mathrm{~mm}$ (Highth). A primary nickel wick has a pore radius of $1.5 \mu \mathrm{m}$ and there is no secondary wick. A bayonet is used to drive the vapor bubbles out of the evaporator core, which is extended to the middle 
position of the evaporator core from the liquid line. For the purpose of convenience, the CC that is not passed through by the bayonet is called $\mathrm{CC} 1$ and the other one is called $\mathrm{CC} 2$. The transport lines are all stainless steel smooth-walled tubes with an outer diameter of $3.0 \mathrm{~mm}$. The condenser tube is welded to several copper plates. These copper plates are contacted thermally with a water cooled heat sink with thermal conductive grease and provide the required surface area for the heat dissipation. Table 1 lists the primary geometrical features of the DCCLHP. In order to reduce the influence of the external air convection, the whole loop was wrapped with thermal insulation materials (Rubber Foam Thermal Insulation Sheet, $0.034 \mathrm{~W} /(\mathrm{m} \cdot \mathrm{K})$ ) and installed in a stainless steel enclosure filled with glass wool.

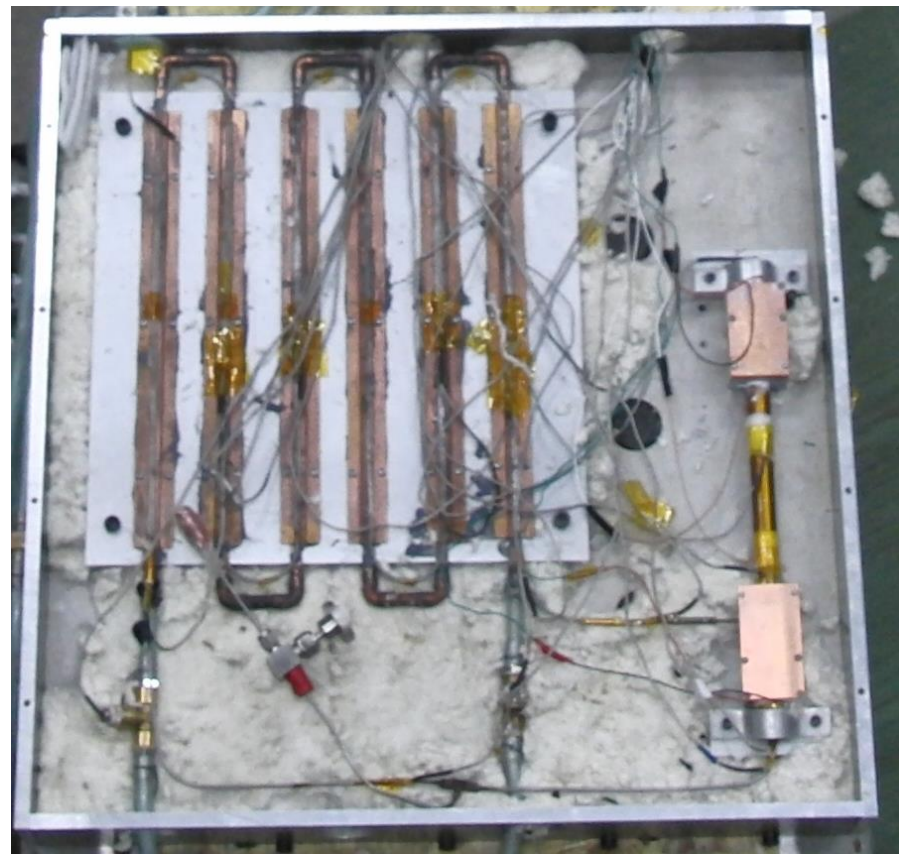

(a) Photo of the DCCLHP

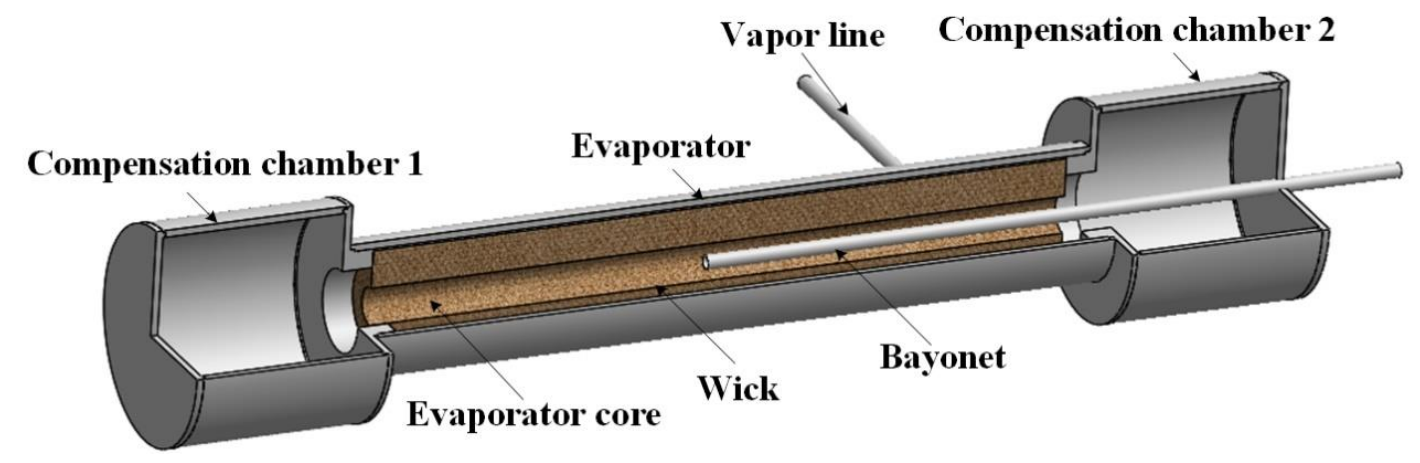

(b) Internal construction of evaporation and CCs

Fig. 1. A photo of the DCCLHP and internal construction of evaporator and CCs. 
Table 1. The primary geometrical features of the DCCLHP

\begin{tabular}{|c|c|c|}
\hline \multirow[t]{5}{*}{ Wick } & Material & Nickel \\
\hline & O.d./i.d. $\times$ Length & $18 \mathrm{~mm} / 6 \mathrm{~mm} \times 190 \mathrm{~mm}$ \\
\hline & Pore radius & $1.5 \mu \mathrm{m}$ \\
\hline & Porosity & $55 \%$ \\
\hline & Permeability & $>5 \times 10^{-14} \mathrm{~m}^{2}$ \\
\hline \multirow[t]{2}{*}{ Evaporator } & Material & Stainless steel \\
\hline & O.d./i.d. $\times$ Length of casing & $20 \mathrm{~mm} / 18 \mathrm{~mm} \times 209 \mathrm{~mm}$ \\
\hline \multirow[t]{3}{*}{$\mathrm{CC}$} & Material & Stainless steel \\
\hline & O.d./i.d. $\times$ Length & $27 \mathrm{~mm} / 25 \mathrm{~mm} \times 64 \mathrm{~mm}$ \\
\hline & Number & 2 \\
\hline \multirow[t]{2}{*}{ Vapor line } & Material & Stainless steel \\
\hline & O.d./i.d. $\times$ Length & $3 \mathrm{~mm} / 2.6 \mathrm{~mm} \times 225 \mathrm{~mm}$ \\
\hline \multirow[t]{2}{*}{ Liquid line } & Material & Stainless steel \\
\hline & O.d./i.d. $\times$ Length & $3 \mathrm{~mm} / 2.6 \mathrm{~mm} \times 650 \mathrm{~mm}$ \\
\hline \multirow[t]{5}{*}{ Condenser } & Material of tube & Stainless steel \\
\hline & O.d./i.d. $\times$ Length & $3 \mathrm{~mm} / 2.6 \mathrm{~mm} \times 2200 \mathrm{~mm}$ \\
\hline & Material of plate & Copper \\
\hline & Number of plate & 6 \\
\hline & Length $\times$ Width $\times$ High & $350 \mathrm{~mm} \times 30 \mathrm{~mm} \times 1 \mathrm{~mm}$ \\
\hline
\end{tabular}

\subsection{Experimental apparatus and procedure}

230 In the current work, an experimental apparatus was designed and built to facilitate testing of 231 the DCCLHP under acceleration conditions, as shown schematically in Fig. 2. It is mainly 232 composed of acceleration simulating and control subsystem, data acquisition and control 233 subsystem, water cooling circulation subsystem as well as test section. The detailed descriptions 234 of each subsystem can be referred to Ref $[32,33]$. For the purpose of completeness, a brief introduction of the test rig will be mentioned here. 


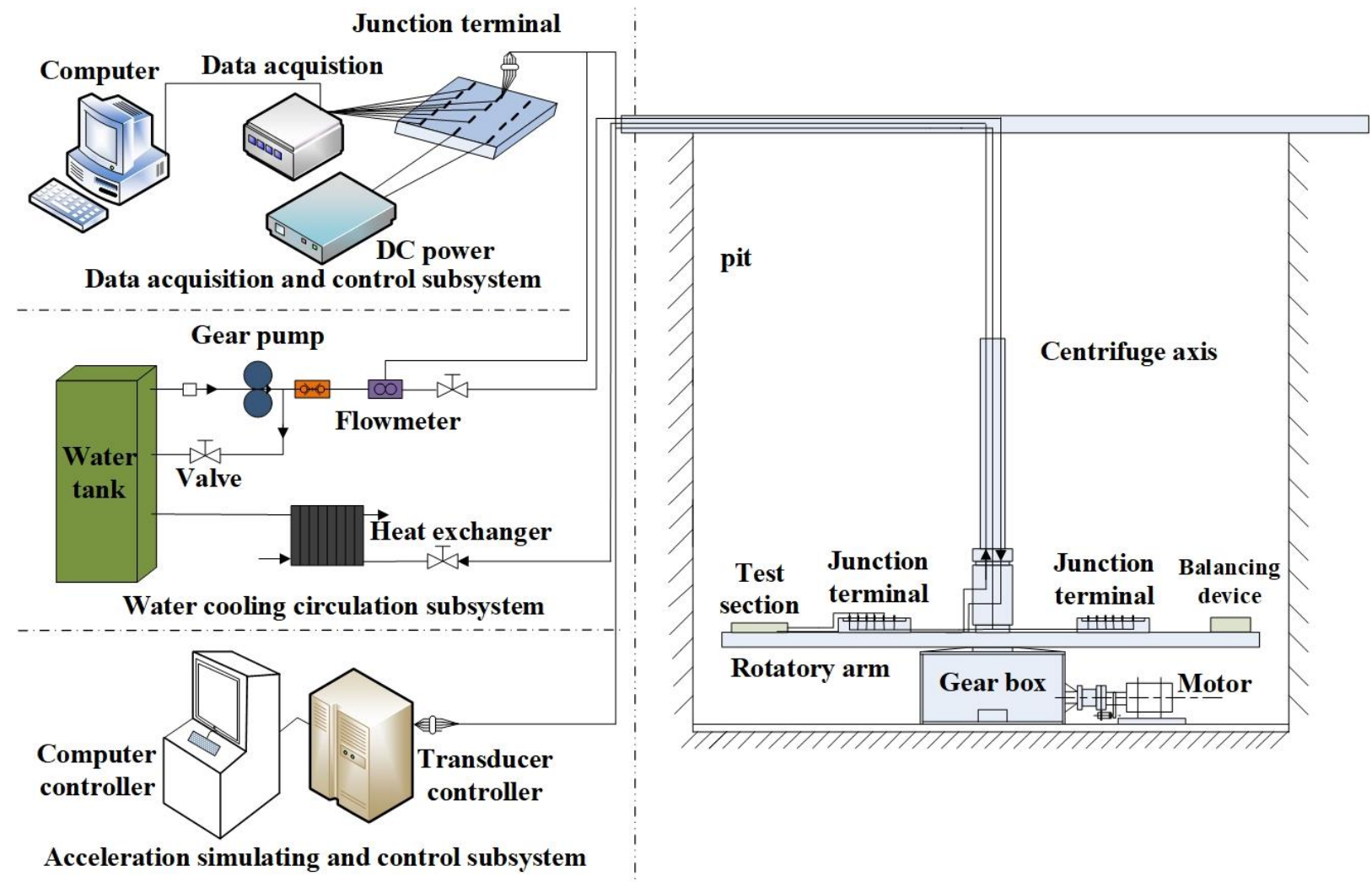

(a) Schematic layout of the experimental apparatus

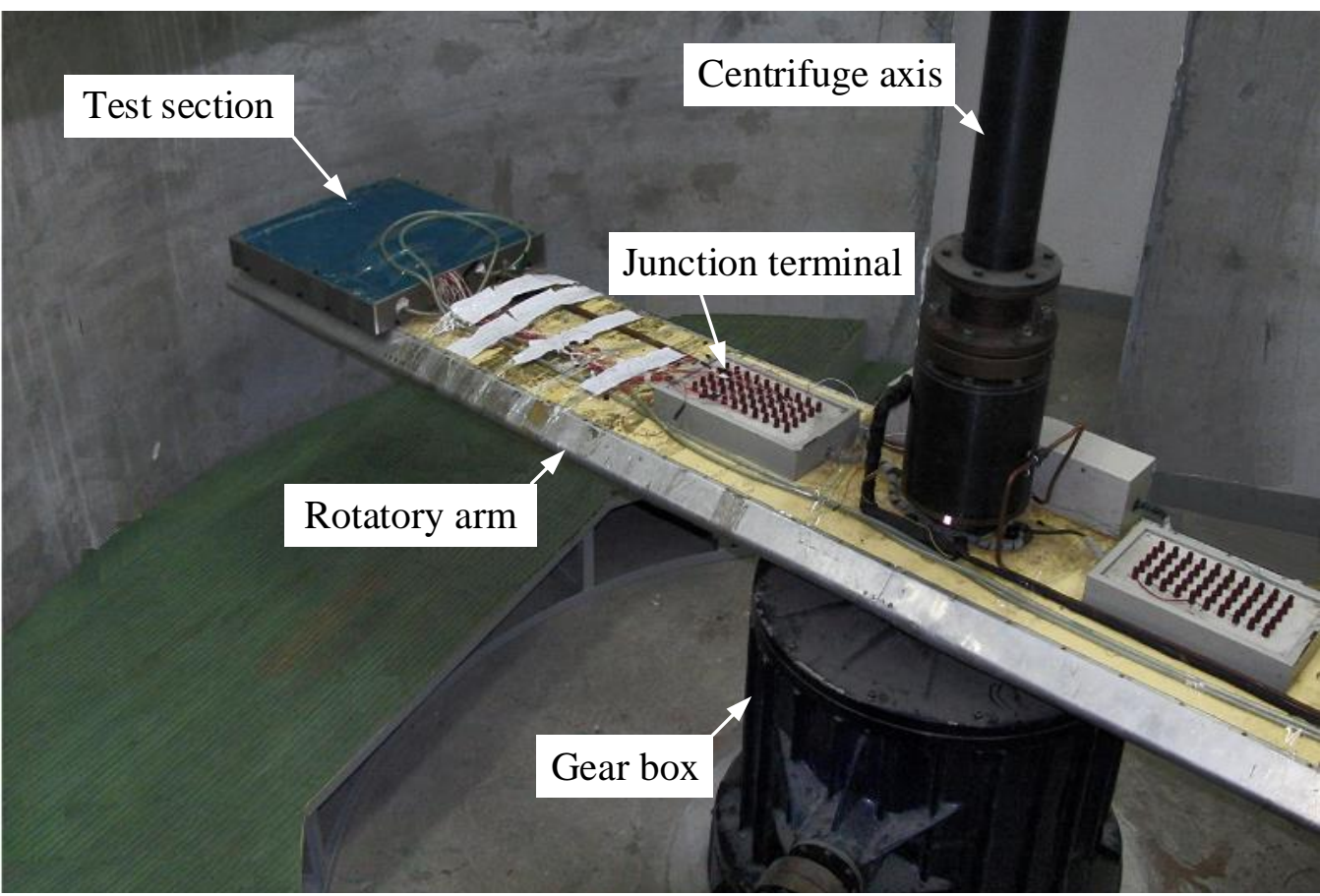

(b) overview photo of the centrifuge and test section

Fig. 2. Schematic layout of experimental apparatus and overview photo of the centrifuge and test section. (a) Schematic layout of experimental apparatus. (b) overview photo of the centrifuge and test section. 
The DCCLHP was placed in horizontal orientation on the rotatory arm, as shown in Fig. 3. The evaporator and CCs were located at the outer edge of the rotatory arm and the axis of the evaporator and CCs was perpendicular to the direction of the radial acceleration. In order to make the acceleration ratio in all the loop suffered fall in the range from $90 \%$ to $130 \%$, which was required by GB/T2423.15, the center of the test article located at the radius of $2.0 \mathrm{~m}$ and the setting value of the rotating radius of the centrifuge was set to $1.9 \mathrm{~m}$. The continuous operation of the centrifuge for no more than an hour was required due to safety concerns.

A flexible polyimide film electric resistance heater adhesively attached to the outer wall of the evaporator was used to apply heat load on the evaporator. Different heat loads could be produced by adjusting the output current ranged from 0 to $5 \mathrm{~A}$ and voltage of the DC power supply (DH1716A-13) ranged from 0 to $250 \mathrm{~V}$, respectively. The amount of heat applying on the evaporator was transmitted to the cooling water inside the aluminum cold plate (type 6061). In order to maintain the same heat sink temperature, the cooling water at the thermostatic water tank was kept at $19^{\circ} \mathrm{C}$.

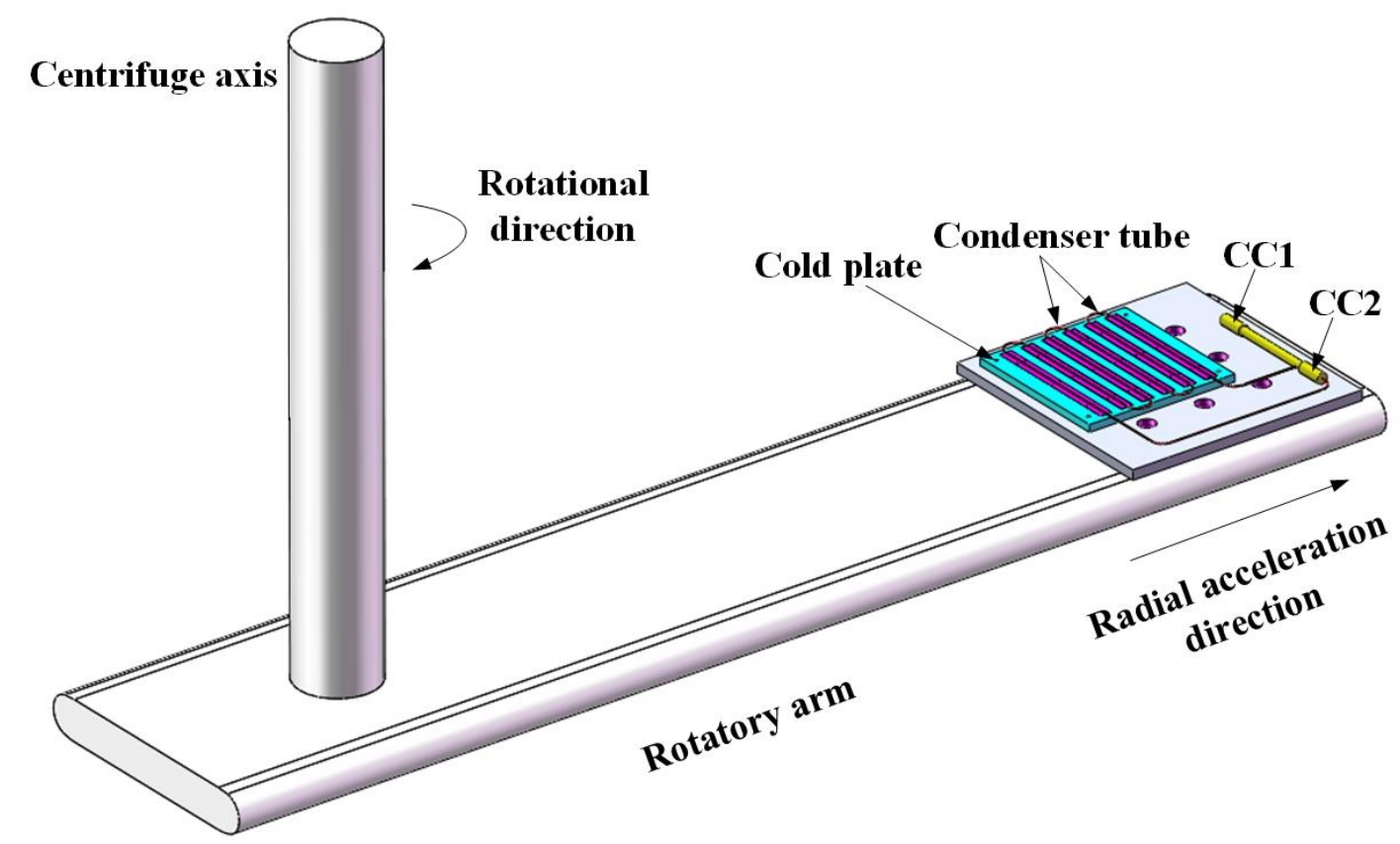

Fig. 3. Configuration of DCCLHP mounted on the centrifuge.

During the test, resistance temperature detectors (RTDs, $\pm 0.06{ }^{\circ} \mathrm{C}$ at $0{ }^{\circ} \mathrm{C}$ ) PT100 were used to monitor the temperature profile of the DCCLHP and the temperature at both inlet and outlet of the cold plate. The position illustration of the RTDs adhered on the outer wall of each component of the DCCLHP is schematically presented in Fig. 4. RTD1 and RTD2 were located at the top of 
the $\mathrm{CC} 1$ and $\mathrm{CC} 2$ outer surface, respectively. RTD3 was attached on the evaporator, while RTD4 was closed to the outlet of the vapor line. RTD5 and RTD6 were located at the middle position of the U shape bend of the condenser, respectively. RTD7, RTD8 and RTD9 were placed at the inlet, middle and outlet of the liquid line. RTD10 and RTD11 were used to measure the temperatures of the cooling water at both inlet and outlet of the cold plate. RTD12 was used to monitor the surrounding ambient temperature. Four-wire system of these RTDs was utilized to measure the temperature. Moreover, calibration of RTDs was conducted by the thermostatic water bath method prior to the real experiment. For the surrounding ambient temperature and the cooling water temperature at both inlet and outlet of the cold plate, the range of 16 to $30{ }^{\circ} \mathrm{C}$ with $2{ }^{\circ} \mathrm{C}$ intervals was expected to calibrate the corresponding RTDs. While for the loop temperature, the prospective range of $18-60{ }^{\circ} \mathrm{C}$ in $2{ }^{\circ} \mathrm{C}$ intervals was used to calibrate the corresponding RTDs.

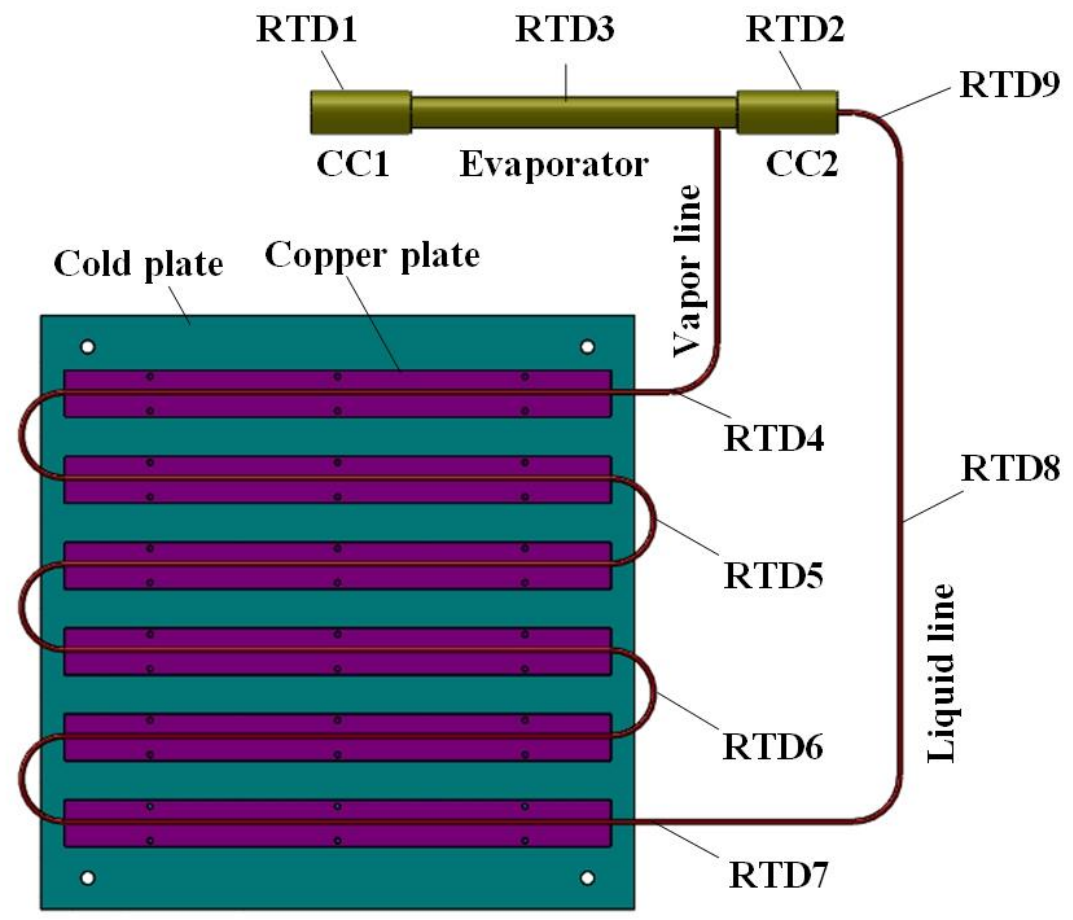

Fig. 4. Schematic location of RTDs along the loop.

When starting the real experiment, the cooling water firstly circulates until the entire system reaches to a steady state. The start-up time of the centrifuge is set to $30 \mathrm{~s}$ which required for the acceleration to reach a set value. Then the heat load or the acceleration load was applied. In the current work, there are two different loading modes. The loading mode I refers to the heat load is firstly applied on the evaporator and then the acceleration load is applied when the DCCLHP operates to a steady state under terrestrial gravity. On the other hand, the loading mode II stands 
for both the heat load and acceleration load are applied at the same time. For the purpose of comparisons, a series of tests were firstly conducted under terrestrial gravity to obtain the basic operating performance of the DCCLHP. Afterwards, the above two loading modes are used to study the effect of the acceleration magnitude and heat load on the operating performance of the DCCLHP under acceleration fields. There are totally six various radial acceleration magnitudes $\left(a_{\mathrm{r}}=1 \mathrm{~g}, 3 \mathrm{~g}, 5 \mathrm{~g}, 7 \mathrm{~g}, 9 \mathrm{~g}, 11 \mathrm{~g}\right)$ and six heat loads $\left(Q_{\mathrm{e}}=25 \mathrm{~W}, 80 \mathrm{~W}, 150 \mathrm{~W}, 200 \mathrm{~W}, 250 \mathrm{~W}, 300\right.$ W) implemented in the current test. It should be noted that the gravity is always at work in all experiments. The maximum continuous operating time of the centrifuge can not exceed $1 \mathrm{~h}$ for the safety issue. The surrounding ambient temperature in the test room was kept at around 26.0 ${ }^{\circ} \mathrm{C}$ by air conditioning. The cooling water temperature at the inlet of the cold plate was maintained from 19.8 to $21.3^{\circ} \mathrm{C}$.

\section{Experimental results and discussion}

The following sections mainly present the experimental data under both terrestrial gravity and acceleration fields for the purpose of comparison. The operating characteristics of the DCCLHP under terrestrial gravity will be firstly described. Then the operating characteristics of the DCCLHP subjected to acceleration force assisted are shown at loading mode I and loading mode II, respectively. Finally, the temperature control performance of the DCCLHP subjected to acceleration force assisted will be analyzed in detail.

\subsection{Operating performance under terrestrial gravity field}

For the purpose of understanding the operating performance of the DCCLHP under terrestrial gravity field, the experimental data of the steady state operating conditions at horizontal position are shown in Fig. 5. The temperatures at different locations of the entire loop are presented. Noting that the DCCLHP cannot reach a steady state within $1 \mathrm{hr}$ under both $25 \mathrm{~W}$ and $80 \mathrm{~W}$ conditions, the maximum temperauters of the evaporator and the other components are used in Fig. 5. 


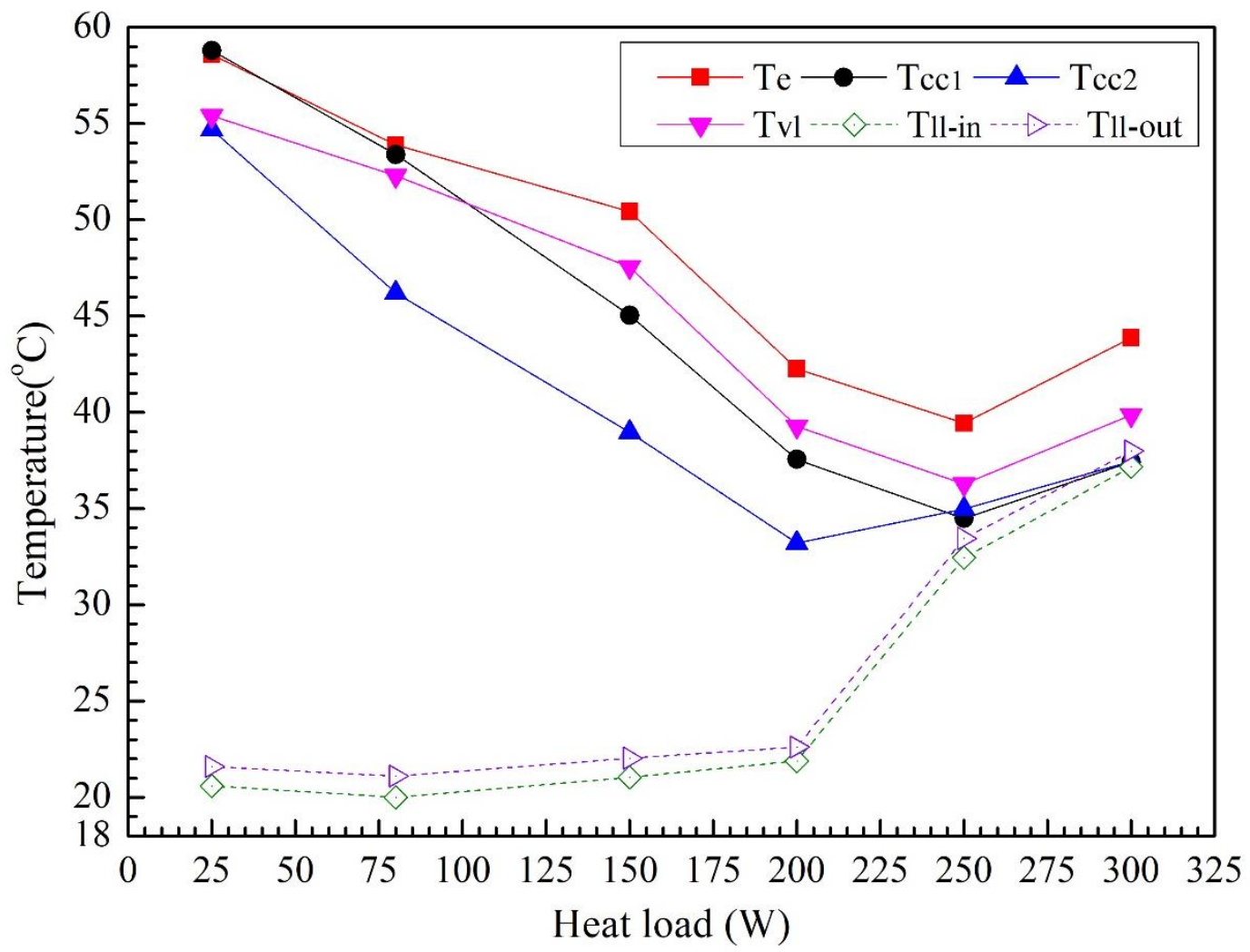

307

Fig. 5 Loop temperatures vs heat load under terrestrial gravity

It can be clearly seen from Fig. 5 that the operating temperature of the DCCLHP shows a typical "V-shape" trend as the evaporator temperature against heat load $[2,23]$. When the heat load is below $250 \mathrm{~W}$, the DCCLHP operates at variable conductance mode (VCM). The temperature of $\mathrm{CC} 1$ is greater than that of $\mathrm{CC} 2$, which is caused by the cooling effect of the returning liquid. The temperatures at the inlet and outlet of the liquid line show small variation with the increase of the heat load. When the heat load is equal or greater than $250 \mathrm{~W}$, it operates at the constant conductance mode (CCM), the temperatures of both CCs are nearly the same and close to the temperature of the liquid line, which all ascend with the increase of the heat load.

Fig. 6 depicts the temperature profiles of the loop with time at $25 \mathrm{~W}$ under terrestrial gravity. As can be seen from Fig. 6, the temperatures of the evaporator, vapor line and CCs show a consistent increase to its maximum value followed by a nearly steady value. The temperatures of the liquid line are kept almost constant. After the heat load is applied at $50 \mathrm{~s}$, the evaporator temperature rises from $24.9{ }^{\circ} \mathrm{C}$ to $25.2{ }^{\circ} \mathrm{C}$. Simutaneously, the temperature at the outlet of the vapor line augments from $24.5^{\circ} \mathrm{C}$ to $24.8{ }^{\circ} \mathrm{C}$. The inlet temperature of the liquid line drops gradually. It indicates that the positive flow circulation is established in the loop and the DCCLHP starts up. From $84 \mathrm{~s}$ to $384 \mathrm{~s}$, the temperatures of the evaporator, vapor line and CC1 remain 
almost constant.

After $384 \mathrm{~s}$, the temperatures of the loop except for the liquid line augment again. There is the same level of the liquid in the CCs and core, which determines their thermal link under terrestrial conditions. Due to the cooling effect of the returning liquid, the $\mathrm{CC} 2$ temperautre is lower than that of the $\mathrm{CC} 1$. With the increase of the evaporator temperature, the heat leak from the evaporator to the CCs increases. On the other hand, the temperature difference between the evaporator and CCs keeps almost unchange after approximate 1400 s. In accordance with the Clausis-Clapeyrong equation, the pressure difference between inside and outside wick also maintains constant.

$$
\Delta p=\left(\frac{d p}{d T}\right)_{\mathrm{sat}} \cdot \Delta T=\frac{h_{\mathrm{vg}}}{T v_{\mathrm{vg}}} \cdot \Delta T
$$

where $h_{\mathrm{vg}}$ is the heat of vaporization of the working fluid, $v_{\mathrm{vg}}$ is the difference in the vapor and liquid specific volumes, $\Delta p$ is equal to the total pressure drop of the loop minus the pressure drop through the wick, $\Delta T$ is the temperature difference between the evaporator and the CC.

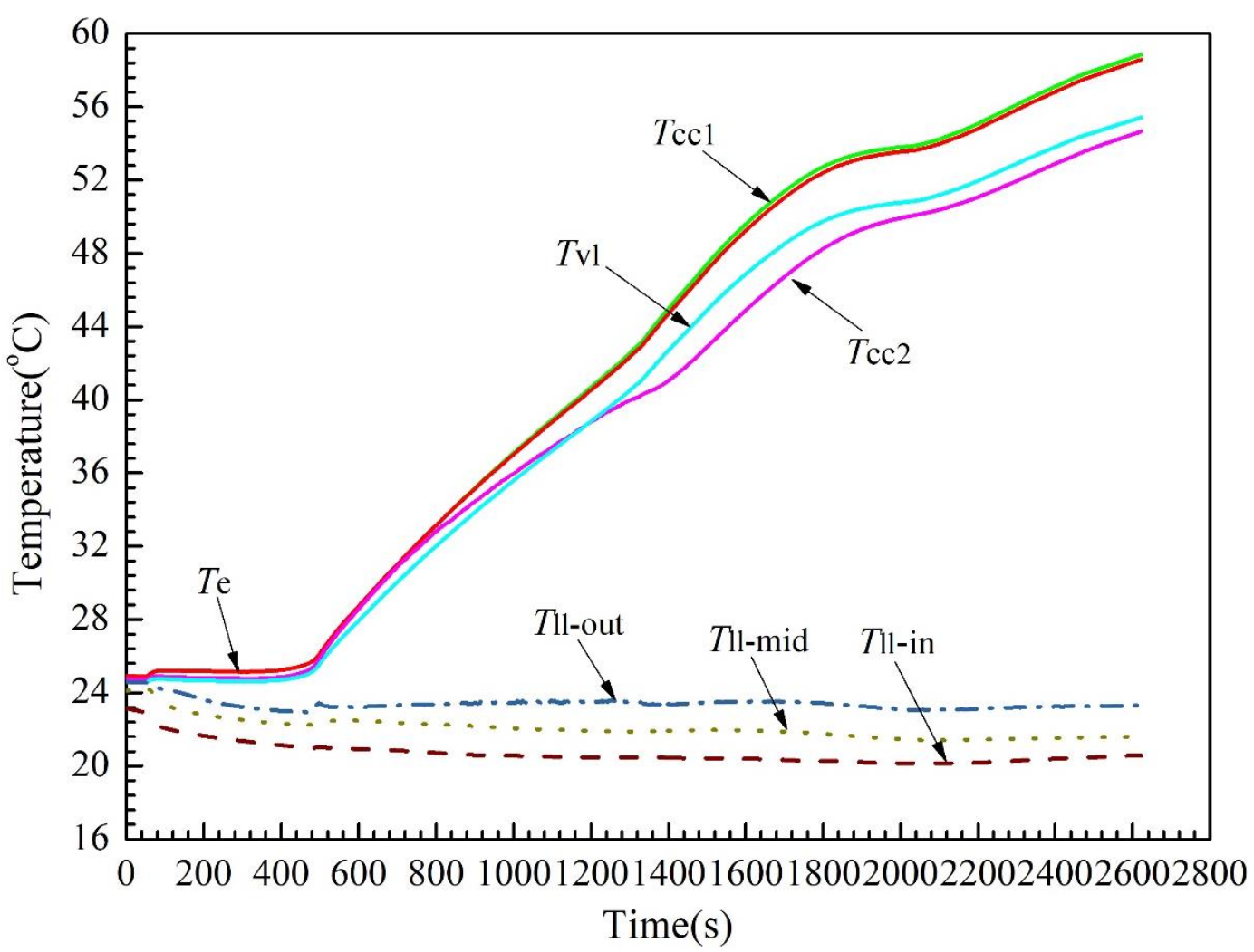

Fig. 6. Temperature profiles of the loop with time at $25 \mathrm{~W}$ under terrestrial gravity.

For a given heat load, the sink temperature and ambinet temperature, the length of the vaporliquid two-phase zone in the condenser would not change and the mass flow rate in the loop is kept constant. Thus, the subcooling of the returning liquid does not change. As a consequence, 
the cooling capacity of the returning liquid is not able to balance the heat leakage from the evaporator to the CCs. The thermal equlibrium can not reach for the DCCLHP.

The condenser is not fully used in terms of the inlet temperature of the liquid line. Because the evaporator temperature reaches $58.6^{\circ} \mathrm{C}$ at $2622 \mathrm{~s}$, the heat load is removed as taking the safety into consideration.

\subsection{Operating performance at loading mode I}

For the loading mode I, the DCCLHP firstly operates to a steady state under terrestrial gravity. Then the acceleration force is applied and a new steady state is achieved. Fig. 7 presents the steady state temperatures of each element of the DCCLHP at different heat loads under $1 \mathrm{~g}, 3 \mathrm{~g}$, $5 \mathrm{~g}$ and $7 \mathrm{~g}$ conditions. From Fig. 7, it can be found that the operating behavior shows a "V-shape" curve at a small acceleration magnitude, whereas it shows a "/-shape" curve at a large acceleration magnitude. Under different acceleration conditions, the $\mathrm{CC} 1$ temperature is lower than that of the CC2. With the increase of the acceleration, the temperature difference between each element of the DCCLHP becomes small. Especially for a small heat load, the temperatures of the vapor line, CCs and liquid line are very close to each other.

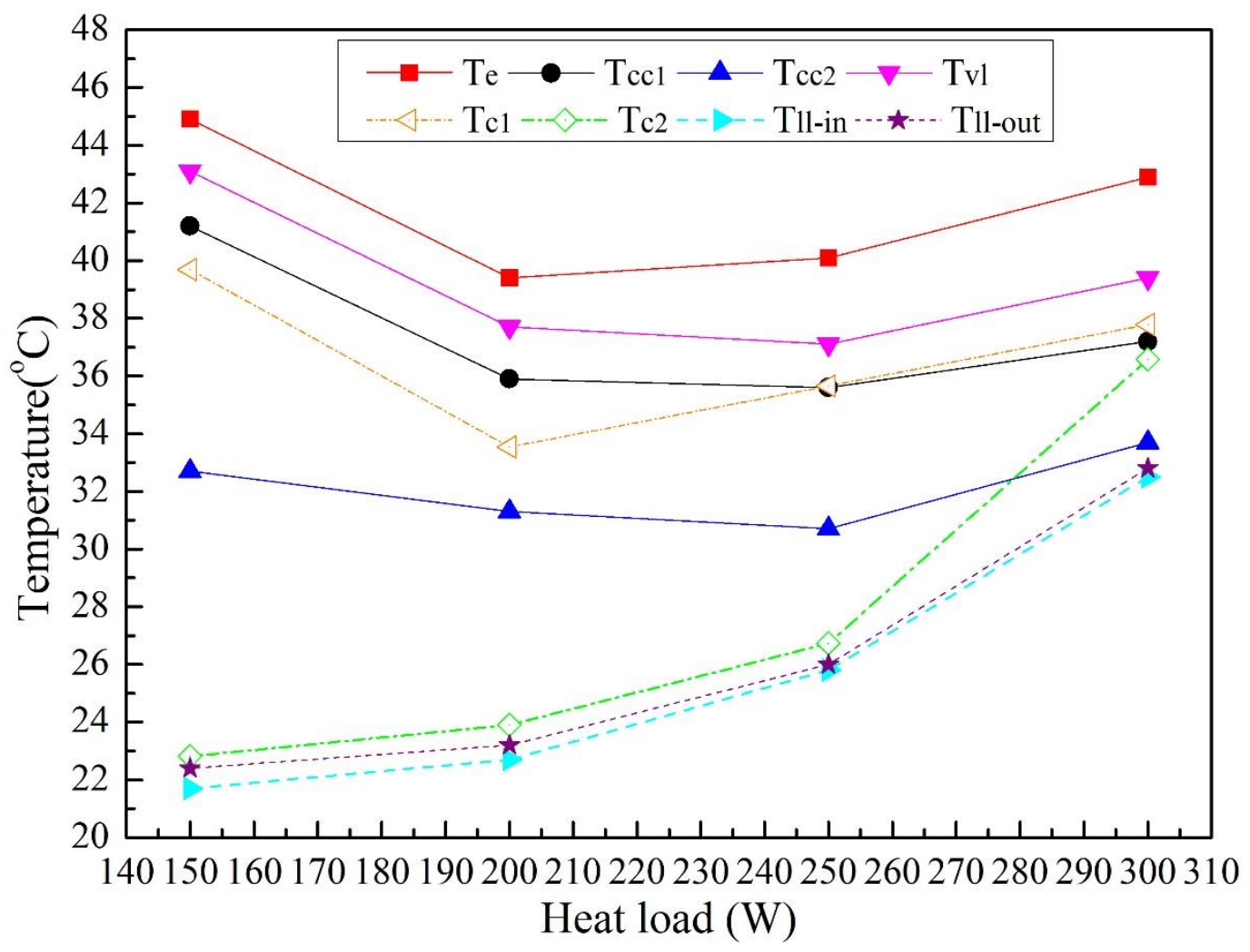

(a) $1 \mathrm{~g}$ 


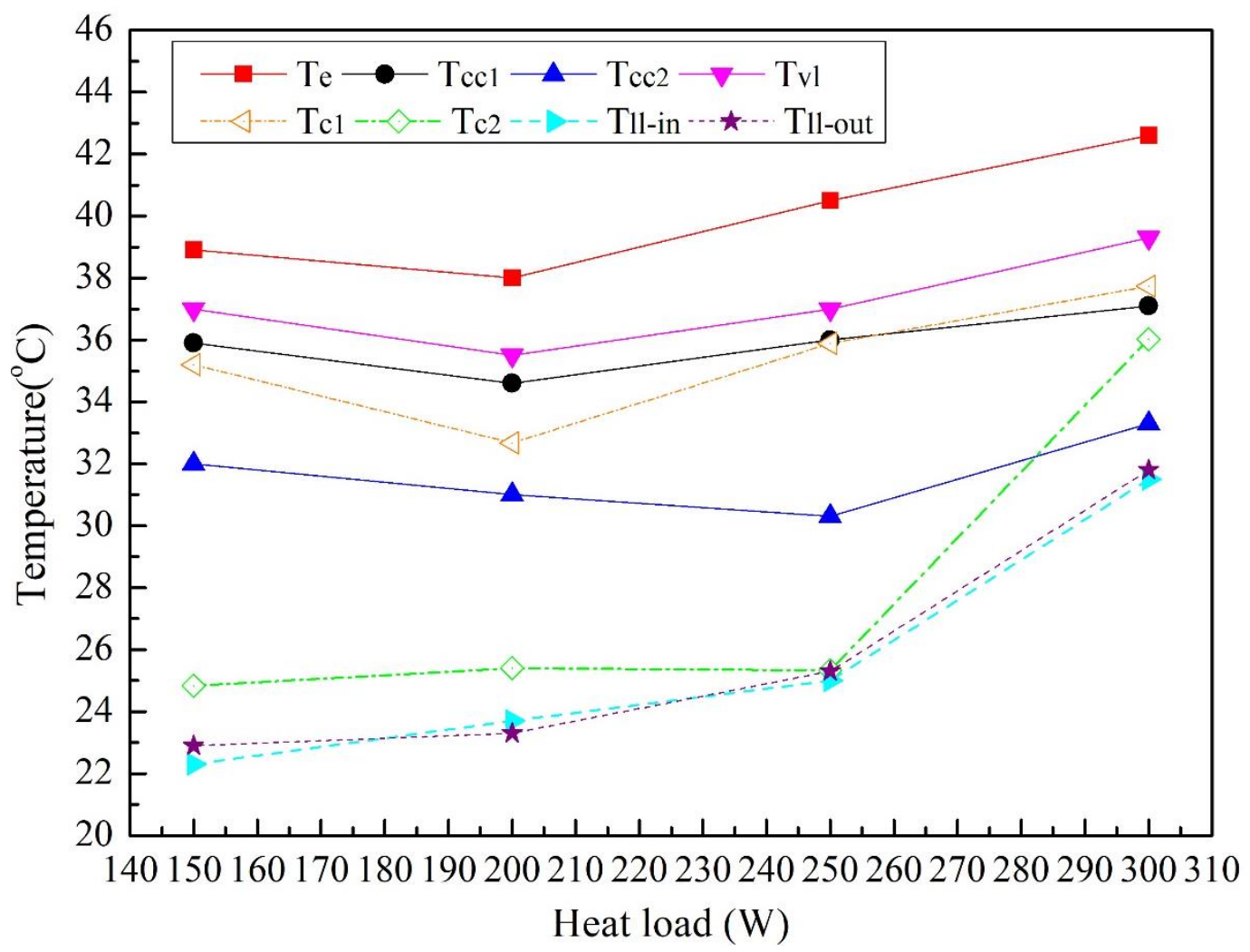

359

(b) $3 \mathrm{~g}$

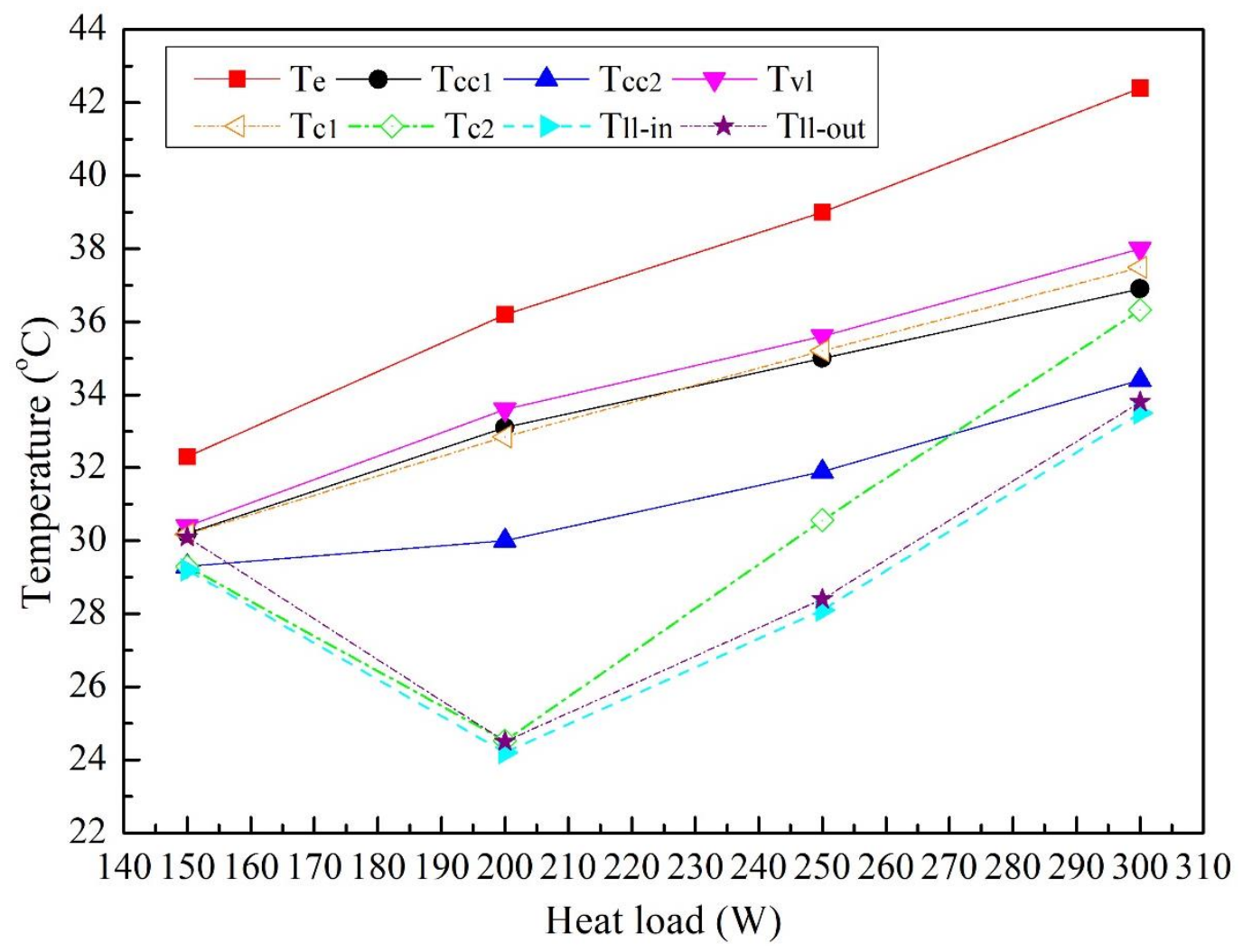

(c) $5 \mathrm{~g}$ 


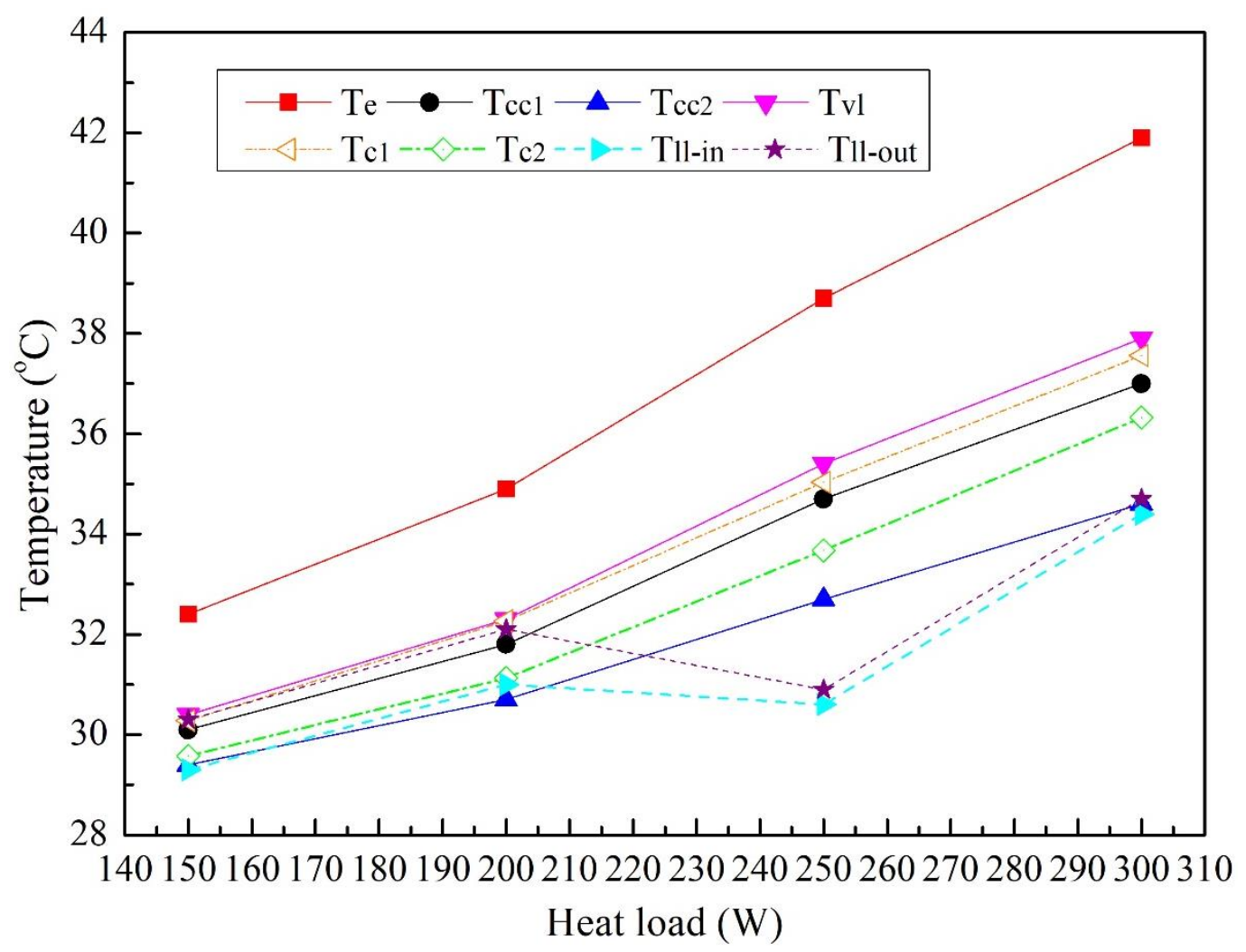

(d) $7 \mathrm{~g}$

Fig. 7. Steady state operating temperature of the loop vs heat load at loading mode I under 1

$$
\text { g, } 3 \text { g, } 5 \text { g and } 7 \text { g conditions. (a) } 1 \text { g. (b) } 3 \text { g. (c) } 5 \text { g. (d) } 7 \text { g. }
$$

As demonstrated in Fig. 7(a), when the heat load is $150 \mathrm{~W}$, the temperatures at the inlet and outlet of the liquid line are close to the sink temperature. The cooling effect of the returning liquid results in the temperature of the $\mathrm{CC} 2$ far below that of the $\mathrm{CC} 1$. As the heat load increases to 200 $\mathrm{W}$, the mass flow rate of the working fluid inside the loop is larger than that at $150 \mathrm{~W}$. The outlet temperature of the liquid line is slightly higher than that at $150 \mathrm{~W}$. Thus, the subcooling of the returning liquid increases. This can balance more heat leak from the evaporator to the CCs. Consequently, the temperatures of the evaporator and CCs drop. When the heat load increases to $200 \mathrm{~W}$ or even higher to $300 \mathrm{~W}$, the subcooling of the returning liquid is difficult to balance the extra heat leak. The temperatures of the $\mathrm{CC} 1$ and evaporator will rise. Under $1 \mathrm{~g}$ condition, the effect of the acceleration does not obviously alter the "V-shape" trend of the steady state operating temperature.

In Fig. 7(b), as the acceleration magnitude increases to $3 \mathrm{~g}$, the "V-shape" trend of the operating temperature becomes unobvious. The evaporator temperature at $150 \mathrm{~W}$ drops to be close to that at 200 W. Compared Fig. 7(b) to Fig. 7(a), the temperature difference among the evaporator, 
vapor line and CCs becomes smaller at $150 \mathrm{~W}$ and $200 \mathrm{~W}$. While the temperature difference shows almost no change for the cases of $250 \mathrm{~W}$ and $300 \mathrm{~W}$. When the acceleration magnitude increases to $5 \mathrm{~g}$, the "V-shape" trend changes to the "/-shape" trend completely, as shown in Fig. 7(c). It should be noted that the temperature difference among the CCs, vapor line and liquid line is approximate within $1^{\circ} \mathrm{C}$ at $150 \mathrm{~W}$. While the temperature distributions of the evaporator, $\mathrm{CCs}$, vapor line and liquid line at each heat load except for $150 \mathrm{~W}$ are similar with those under $1 \mathrm{~g}$ and $3 \mathrm{~g}$ conditions. The evaporator temperature at $150 \mathrm{~W}, 200 \mathrm{~W}$ and $250 \mathrm{~W}$ is lower than that at the same heat load under $3 \mathrm{~g}$ conditions. As the acceleration magnitude is $7 \mathrm{~g}$ shown in Fig. 7(d), the same trend of the operating temperature as the case of $5 \mathrm{~g}$ exhibits. It is at $150 \mathrm{~W}$ and $200 \mathrm{~W}$ that the temperatures of the CCs, vapor line and liquid line show a small difference. The temperature distributions of the loop components at $250 \mathrm{~W}$ and $300 \mathrm{~W}$ are similar with those under $1 \mathrm{~g}, 3 \mathrm{~g}$ and $5 \mathrm{~g}$ conditions.

From the above experimental data, a unique phenomenon is revealed as the DCCLHP operates in a large acceleration field. The operation of the DCCLHP subjected to acceleration force is a bit similar to that with gravity assist under the terrestrial gravity conditions [15, 22]. In the current work, a new operating mode of the DCCLHP, namely, centrifugal force-dominated mode is proposed. For a small heat load and large acceleration, the DCCLHP can operate at the centrifugal force-dominated mode, in which the flow circulation of the working fluid is only driven by the centrifugal force. In other words, the flow circulation in the loop is driven by the net pressure difference from liquid head by the effect of the centrifugal acceleration. Thus, significant deviation from the "V-shape" curve of the operating temperature is caused by the effect. The detailed descriptions on the physical mechanism of the centrifugal force-dominated operation will be discussed in section 3.4.

Fig. 8 depicts the steady state operating temperature and thermal conductance of the DCCLHP at loading mode I. Here, the thermal conductance of the DCCLHP was determined by the heat load on the evaporator, the evaporator temperature and the average temperature of the cold plate:

$$
G=\frac{Q_{\mathrm{e}}}{T_{\mathrm{e}}-\bar{T}_{\mathrm{cp}}}
$$

where $\bar{T}_{\text {cp }}=0.5\left(T_{\text {out }}+T_{\text {in }}\right)$ is the average cold plate temperature, $T_{\text {in }}$ and $T_{\text {out }}$ are the temperature at the inlet and outlet of the cold plate, respectively. $Q_{\mathrm{e}}$ is the heat load on the 
evaporator.

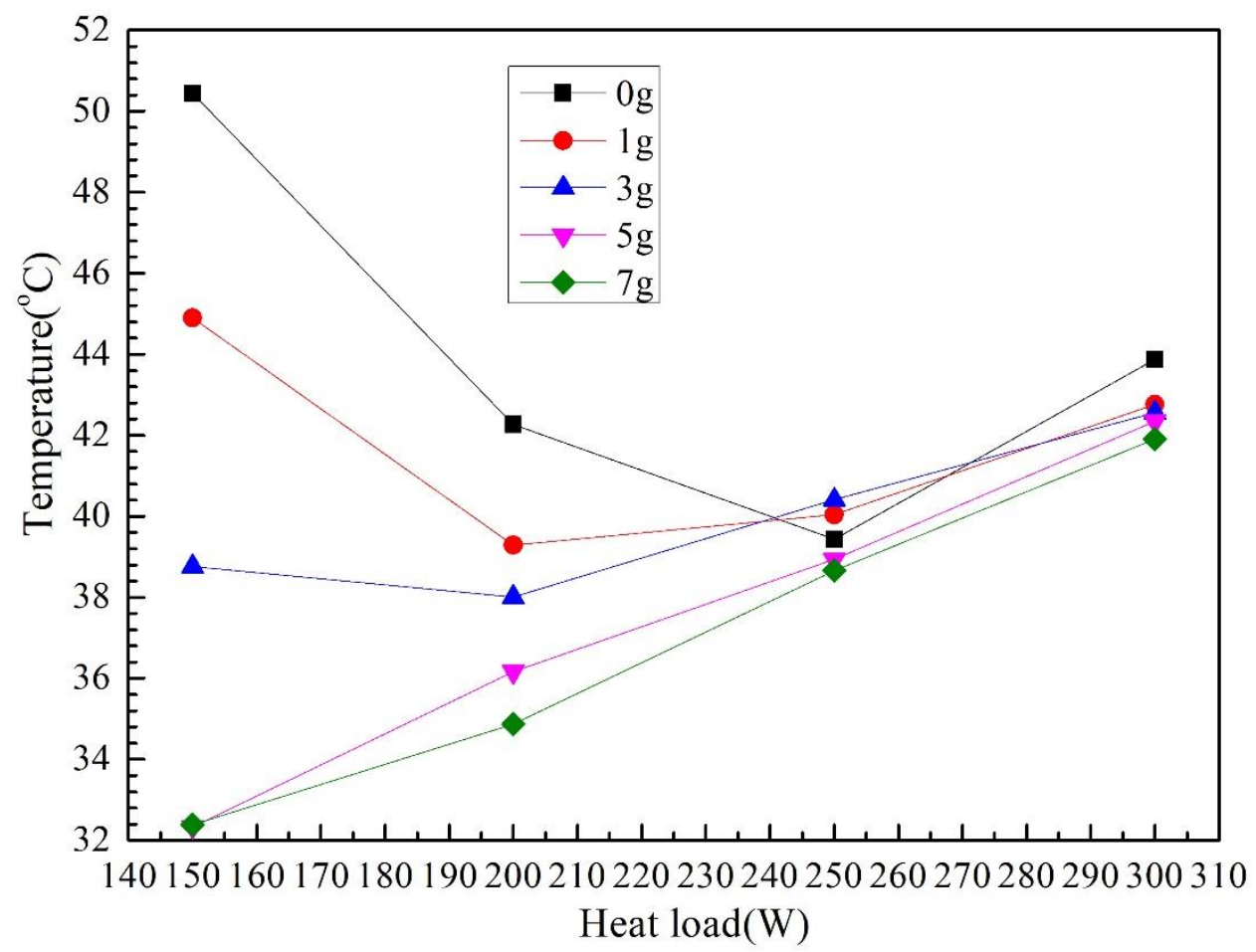

(a) Operating temperature

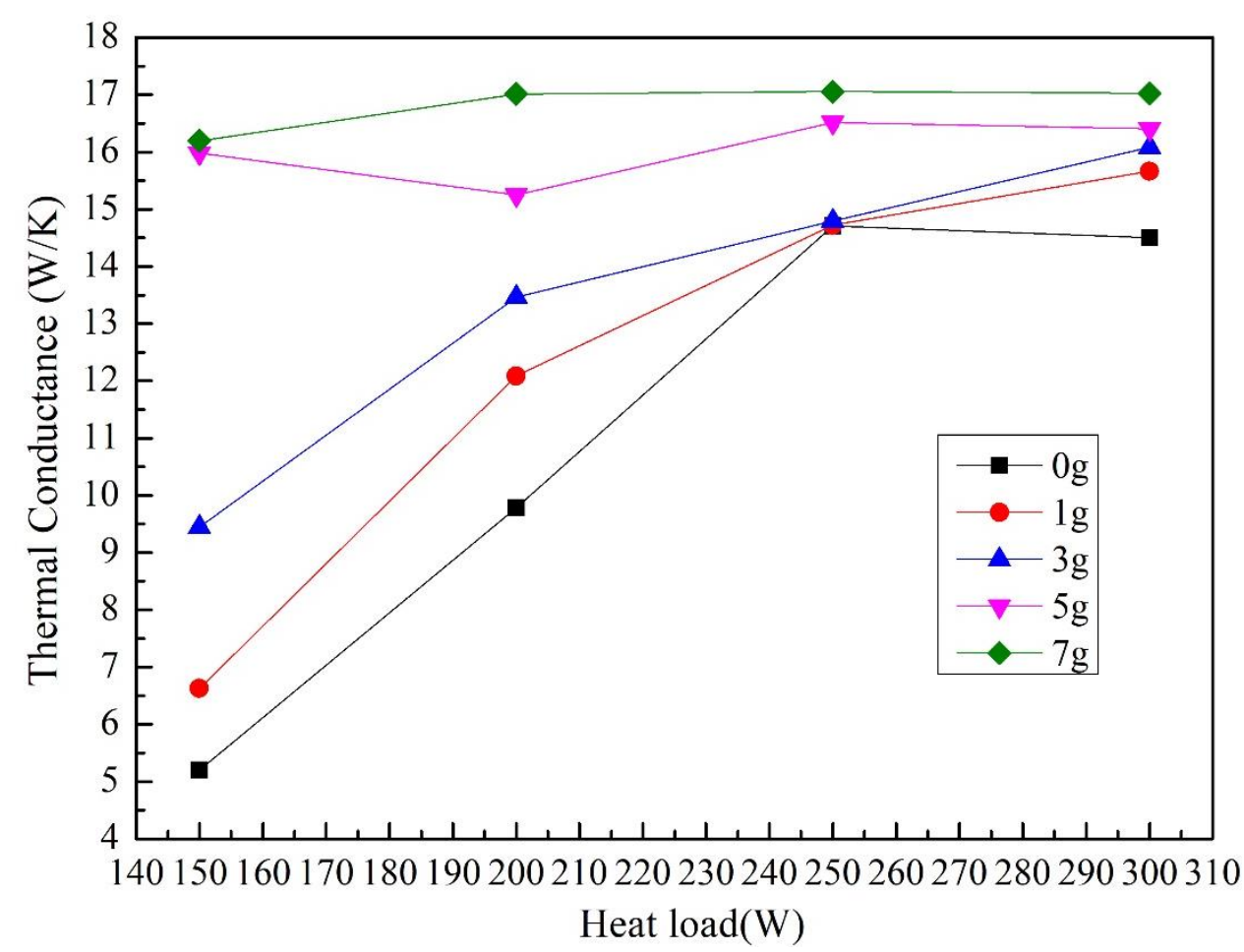

(b) Thermal conductance

Fig. 8 Steady state operating temperature and thermal conductance of the DCCLHP at loading mode I. (a) Operating temperature. (b) Thermal conductance.

417 It can be clearly seen from Fig. 8(a) that the classic "V-shape" curve of the operating temperature with heat load gradually degenerates to "/-shape" oblique line with increasing the 
acceleration. The effect of the acceleration on the operating temperature is more pronounced as the heat load is small. The operating temperature under acceleration conditions is apparently lower than that under terrestrial gravity. In general, with the increase of the acceleration, the operating temperature decreases for each heat load. For the case of smaller heat load, the amount of decrease is greater. For example, the operating temperature at $300 \mathrm{~W}$ under $1 \mathrm{~g}$ to $7 \mathrm{~g}$ conditions ranges from $41.6^{\circ} \mathrm{C}$ to $42.8{ }^{\circ} \mathrm{C}$, whereas at $150 \mathrm{~W}$ it ranges from $32.4{ }^{\circ} \mathrm{C}$ to $45^{\circ} \mathrm{C}$.

From Fig. 8(b), it can be proved that the thermal conductance of the DCCLHP is a function of acceleration magnitude and heat load for a given ambient temperature and sink temperature. The thermal conductance increases with the increase of the acceleration magnitude at a fixed heat load. It should be noted that the variation of the thermal conductance along with the acceleration is more obvious as the heat load is smaller. For the case of terrestrial gravity, the DCCLHP would operate under CCM as the heat load is $250 \mathrm{~W}$ and $300 \mathrm{~W}$, whereas it operates under VCM at 150 $\mathrm{W}$ and $200 \mathrm{~W}$.

However, when the acceleration magnitude is $7 \mathrm{~g}$, the thermal conductance keeps almost unchanged at all heat loads and the operating of the DCCLHP should be referred to CCM. Additionally, according to the results shown in Fig. 7(d), the condenser is fully utilized at $150 \mathrm{~W}$ and $200 \mathrm{~W}$ while most of it is used at $250 \mathrm{~W}$ and $300 \mathrm{~W}$. Thus, the concept of the variable or constant conductance defined for the operating mode of LHP under terrestrial gravity should not be suitable for the case of acceleration. It is suggested that centrifugal force driven mode or capillary-centrifugal force co-driven mode would be used to describe the operation of the DCCLHP.

\subsection{Operating performance at loading mode II}

Compared with the results under terrestrial gravity, the DCCLHP can be able to operate under steady state at $25 \mathrm{~W}$ and $80 \mathrm{~W}$ with loading mode II. Fig. 9 reveals the steady temperature of the loop versus heat load with loading mode II under $3 \mathrm{~g}, 5 \mathrm{~g}, 7 \mathrm{~g}, 9 \mathrm{~g}$ and $11 \mathrm{~g}$, respectively. It can be determined from Fig. 9 that the effect of the acceleration could make the temperature difference between each element of the entire loop become smaller under larger acceleration conditions. Regarding the temperatures of the evaporator, $\mathrm{CCs}$ and vapor line are within a very small range, which are evidently different from those under terrestrial gravity and loading mode I, as shown in Fig. 5 and Fig. 8, respectively. When the heat load is $25 \mathrm{~W}$, the operating 
temperature of the DCCLHP is not more than $26^{\circ} \mathrm{C}$ under acceleration conditions, which is very

450 close to the sink temperature. With the increase of the acceleration, the "V-shape" curve trend of the operating temperature changes to "/-shape" trend with the heat load. Under small acceleration conditions, the temperature of the liquid line deviates far away from that of the other components. Moreover, this deviation is more pronounced at large heat load.

As demonstrated in Fig. 9(a), when the acceleration magnitude is $3 \mathrm{~g}$, the operating temperature shows an obvious "V-shape" curve in the range from $200 \mathrm{~W}$ to $300 \mathrm{~W}$. As the heat load is below $200 \mathrm{~W}$, the operating temperature increases nearly linearly with the heat load. When the heat load changes from $200 \mathrm{~W}$ to $300 \mathrm{~W}$, the operating temperature firstly drops and then rises. According to the temperatures of the condenser, it is clearly observed that a portion of the condenser is used as the heat load is equal or less than $200 \mathrm{~W}$. When the heat load is equal or larger than $250 \mathrm{~W}$, the condenser would not be fully opened in terms of the temperature of the inlet of the condenser and the liquid line. It is at $200 \mathrm{~W}$ that the operating temperature reaches to the maximum value of $40.7^{\circ} \mathrm{C}$ among all the heat loads. At $25 \mathrm{~W}$, it reaches the minimum value of $25.9^{\circ} \mathrm{C}$.

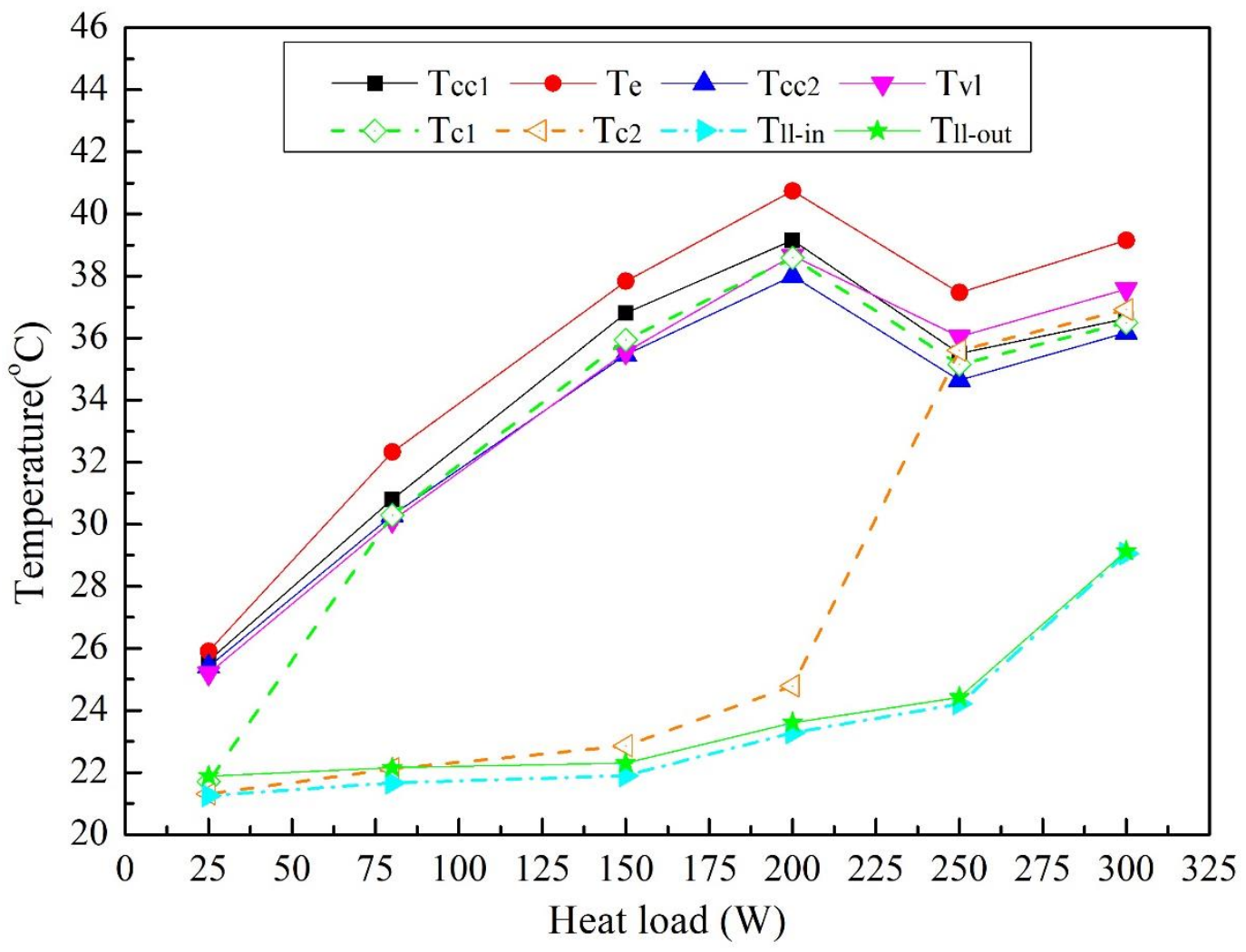




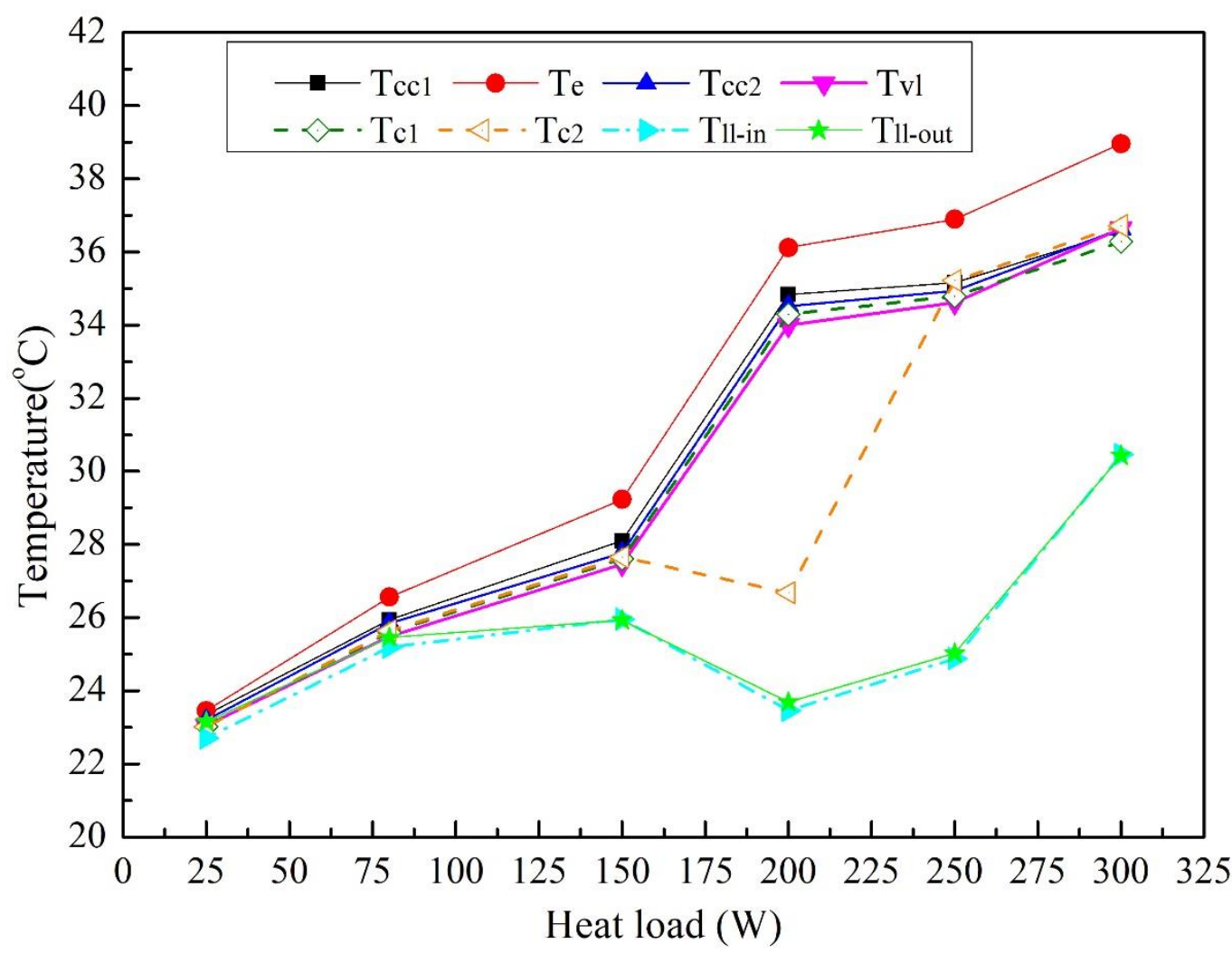

465

(b) $5 \mathrm{~g}$

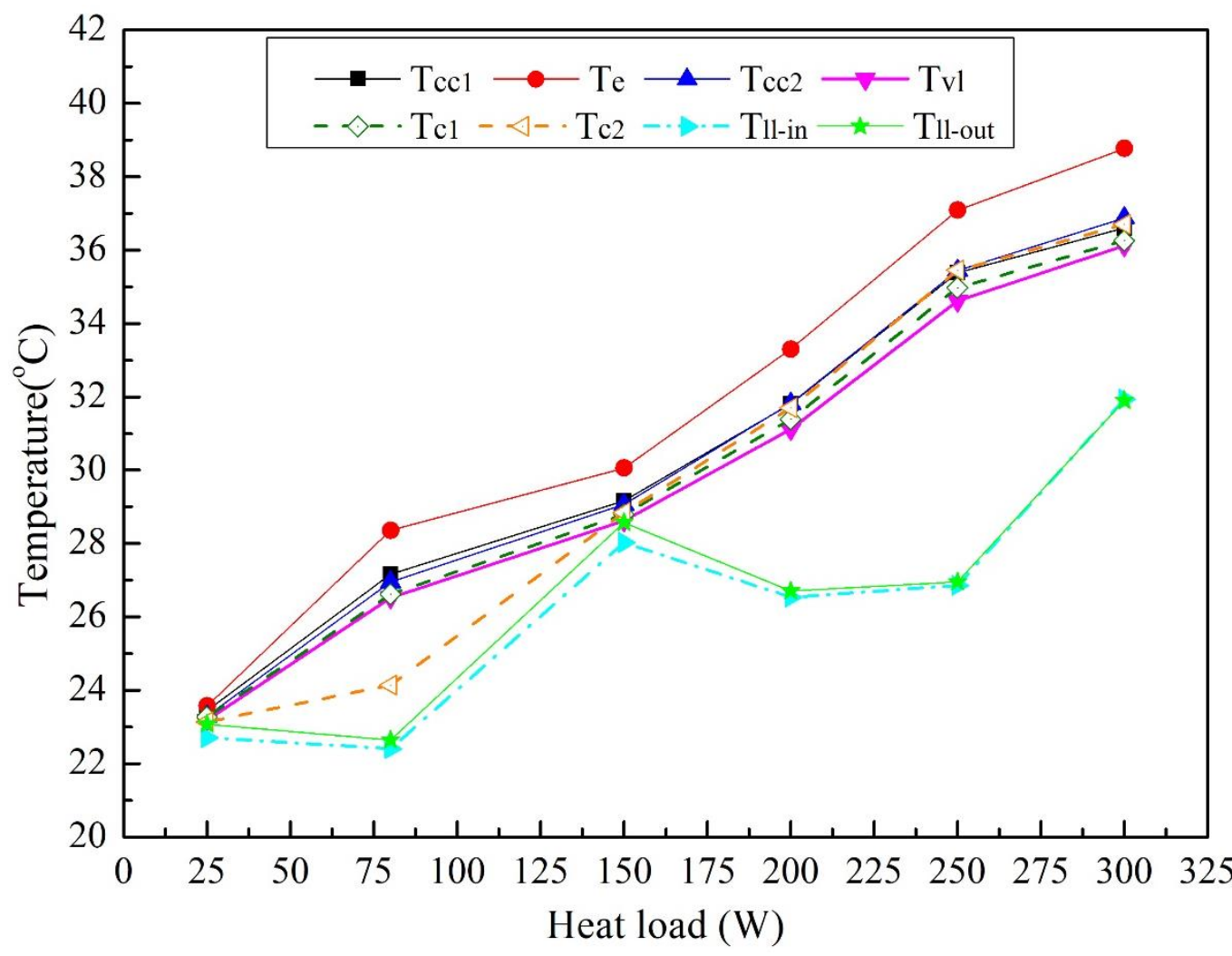

468

(c) $7 \mathrm{~g}$ 


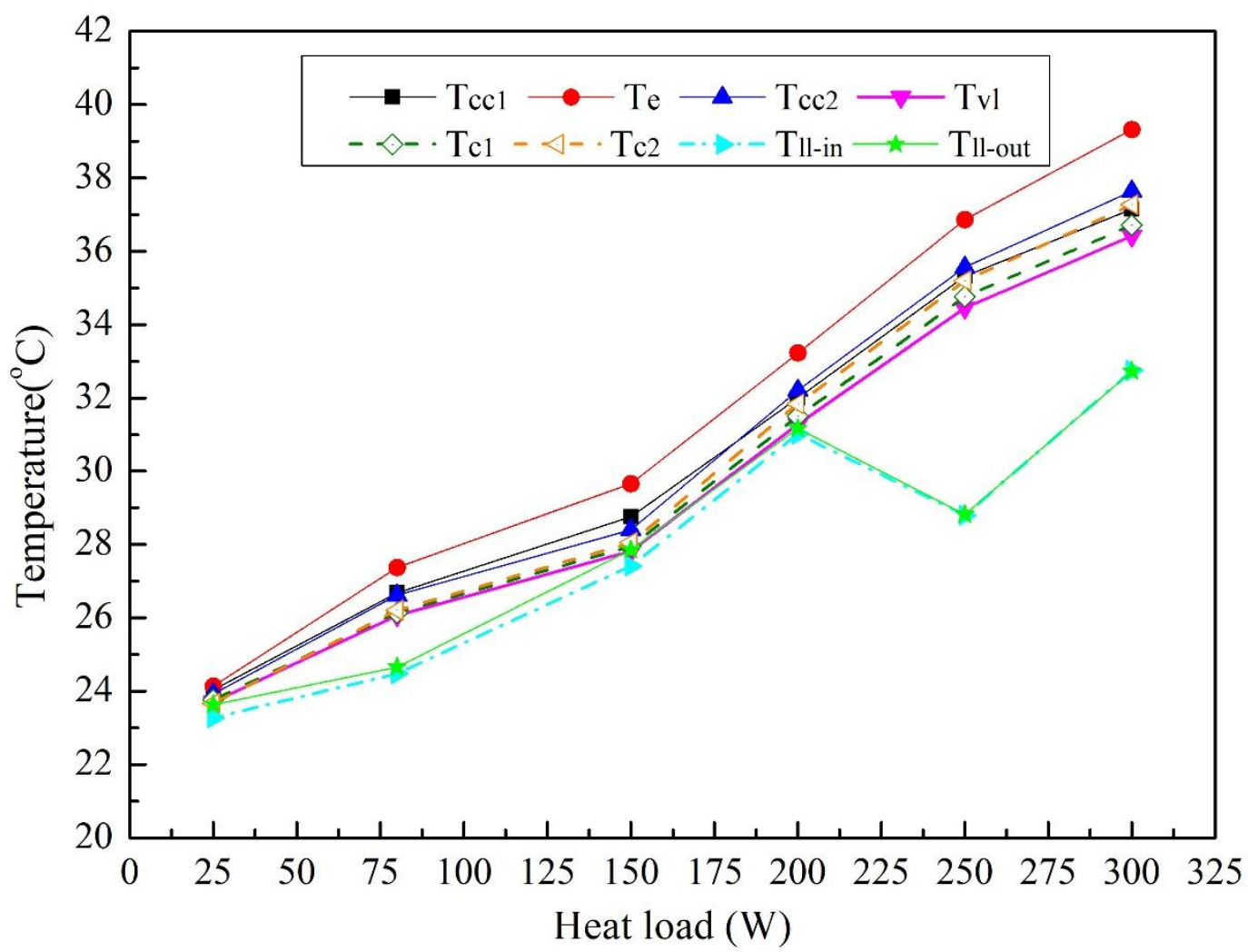

(d) $9 \mathrm{~g}$

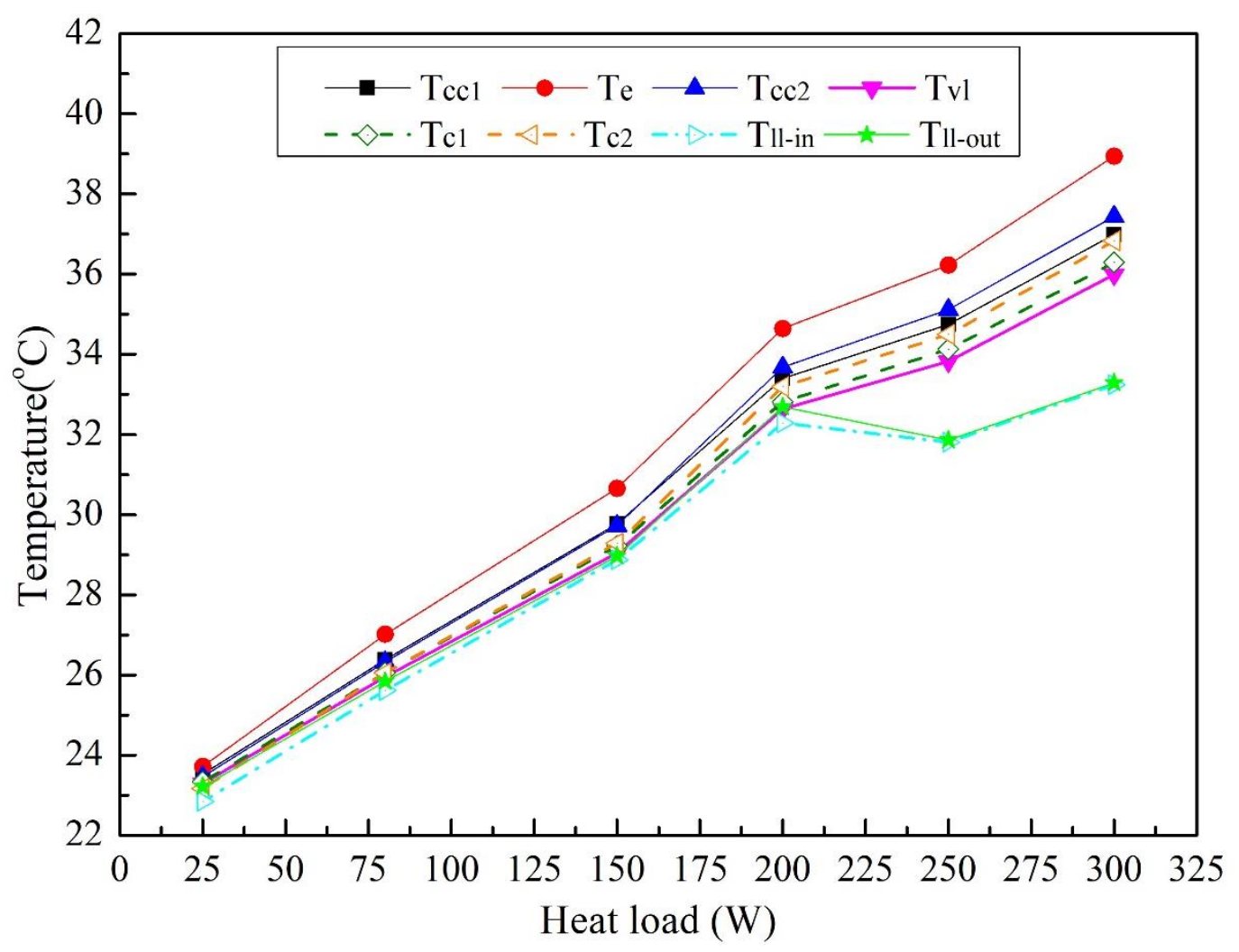

(e) $11 \mathrm{~g}$

Fig. 9 Steady state operating temperature vs heat load at loading mode II under $3 \mathrm{~g}, 5 \mathrm{~g}, 7 \mathrm{~g}, 9 \mathrm{~g}$ and 11g. (a) 3g. (b) 5g. (c) 7g. (d) 9g. (e) 11g. 
When the acceleration increases to $5 \mathrm{~g}$, as shown in Fig. 9(b), the temperatures of the evaporator, vapor line and CCs show a remarkable drop with the heat load of $80 \mathrm{~W}, 150 \mathrm{~W}$ and $200 \mathrm{~W}$. The traditional "V-shape" curve degenerates into a "/-shape" oblique line. As the heat load is $25 \mathrm{~W}$ and $80 \mathrm{~W}$, the temperature difference in the whole loop almost keeps within $1.4{ }^{\circ} \mathrm{C}$. It is expected that the vapor-liquid two-phase flow occurs inside the vapor line and condenser. This phenomenon is similar to that with loading mode I under $150 \mathrm{~W}$ and $5 \mathrm{~g}$ conditions. As a result, the DCCLHP operates at centrifugal force driven mode. As the heat load exceeds $150 \mathrm{~W}$, a portion of the condenser is used. It needs to be noted that the loop temperature oscillates at 150 W. Compared with the case of $3 \mathrm{~g}$, the operating temperature at $200 \mathrm{~W}$ drops to $36.1{ }^{\circ} \mathrm{C}$. At 300 $\mathrm{W}$, the operating temperature reaches to the maximum value of $39.0^{\circ} \mathrm{C}$.

In Fig. 9(c), the effect of the acceleration further reduces the operating temperature at $200 \mathrm{~W}$. While the operating temperature changes a little at the other heat loads. Under $7 \mathrm{~g}$ condition, it is expected that the DCCLHP operates at centrifugal force driven mode at $25 \mathrm{~W}$ and $150 \mathrm{~W}$ with the condenser is fully open. Under the other heat loads, the condenser would not be fully used. In comparison with the case of $5 \mathrm{~g}$ at $80 \mathrm{~W}$, the loop temperature oscillation occurs. The operating temperature increases approximate $1.8{ }^{\circ} \mathrm{C}$. The vapor-liquid interface moves to somewhere between the point 9 and 10 . The operating temperature at $25 \mathrm{~W}$ and $300 \mathrm{~W}$ is $23.6^{\circ} \mathrm{C}$ and 38.8 ${ }^{\circ} \mathrm{C}$, respectively.

When the acceleration is $9 \mathrm{~g}$, as shown in Fig. 9(d), the operating temperature at each heat load changes a little in contrast to that at $7 \mathrm{~g}$. The temperature of the liquid line is closer to the temperature of the vapor line and condenser. The heat load range operating at the centrifugal force driven mode expands to $200 \mathrm{~W}$. A portion of the condenser is used for the heat load $80 \mathrm{~W}, 250 \mathrm{~W}$ and $300 \mathrm{~W}$. Noting that the temperature oscillation occurs as well. The operating temperature at $25 \mathrm{~W}$ and $300 \mathrm{~W}$ is $24.1{ }^{\circ} \mathrm{C}$ and $39.3{ }^{\circ} \mathrm{C}$, respectively.

As shown in Fig. 9(e), as the acceleration magnitude increases to $11 \mathrm{~g}$, the temperatures of the 500 liquid line are closer to the temperature of the vapor line and condenser. The operating temperature shows a slight change comparing with that at $9 \mathrm{~g}$. The heat load range of the centrifugal force driven mode is from $25 \mathrm{~W}$ to $200 \mathrm{~W}$. Furthermore, the entire condenser is used within the heat load range. It should be noted that the temperature oscillation does not occur at $80 \mathrm{~W}$. The operating temperature at $25 \mathrm{~W}$ and $300 \mathrm{~W}$ is $23.7^{\circ} \mathrm{C}$ and $39.0^{\circ} \mathrm{C}$, respectively. 
The steady state operating temperature and thermal conductance at loading mode II are shown in Fig. 10. The experimental data under standard gravity conditions are also presented for the purpose of comparison. It can be clearly seen that the operating temperature under standard gravity is obviously higher than that under acceleration conditions for a fixed heat load. As the heat load is less than $200 \mathrm{~W}$, the operating temperature variation with the heat load shows an opposite trend under between standard gravity and acceleration conditions. With the increase of the acceleration magnitude, the operating temperature decreases generally. The decrease ratio increases with decreasing heat load. Under high acceleration conditions above $5 \mathrm{~g}$, the operating temperature presents an approximately linear increase with the heat load. Additionally, the operating temperature at different acceleration presents a slight change at a fixed heat load. For instance, the operating temperature at $300 \mathrm{~W}$ is in the range from $36.6{ }^{\circ} \mathrm{C}$ to $37.2{ }^{\circ} \mathrm{C}$ as the acceleration magnitude ranges from $3 \mathrm{~g}$ to $11 \mathrm{~g}$. When the acceleration magnitude exceeds a critical value, the operating temperature at a given heat load changes slightly as further increasing the acceleration. Similarly, when the heat load exceeds a critical value, the operating temperature also changes slightly with the acceleration.

From Fig. 10(b), it can be found that the effect of acceleration significantly increases the thermal conductance of the DCCLHP, especially under small heat load and large acceleration conditions. This indicates that the operating temperature is dependent on the acceleration magnitude and heat load. Under large acceleration conditions, the increase of the thermal conductance becomes small. As the acceleration magnitude is $3 \mathrm{~g}$, the thermal conductance increases monotonically with the heat load. The minimum and maximum value is $5.1 \mathrm{~W} / \mathrm{K}$ and $20.8 \mathrm{~W} / \mathrm{K}$, respectively. Under $5 \mathrm{~g}$ condition, the thermal conductance at $200 \mathrm{~W}$ is lower than that at the other heat loads except for $25 \mathrm{~W}$, which is $16.0 \mathrm{~W} / \mathrm{K}$. The reason could be that the operating temperature of the DCCLHP at $200 \mathrm{~W}$ is relatively high, as shown in Fig. 10(a). Furthermore, the high operating temperature should be resulted from the effect of the $5 \mathrm{~g}$ acceleration. According to Eq. (2), the thermal conductance at $200 \mathrm{~W}$ is lower than that at $150 \mathrm{~W}$ and $250 \mathrm{~W}$ when the average temperature of the cold plate is close for these three cases. 


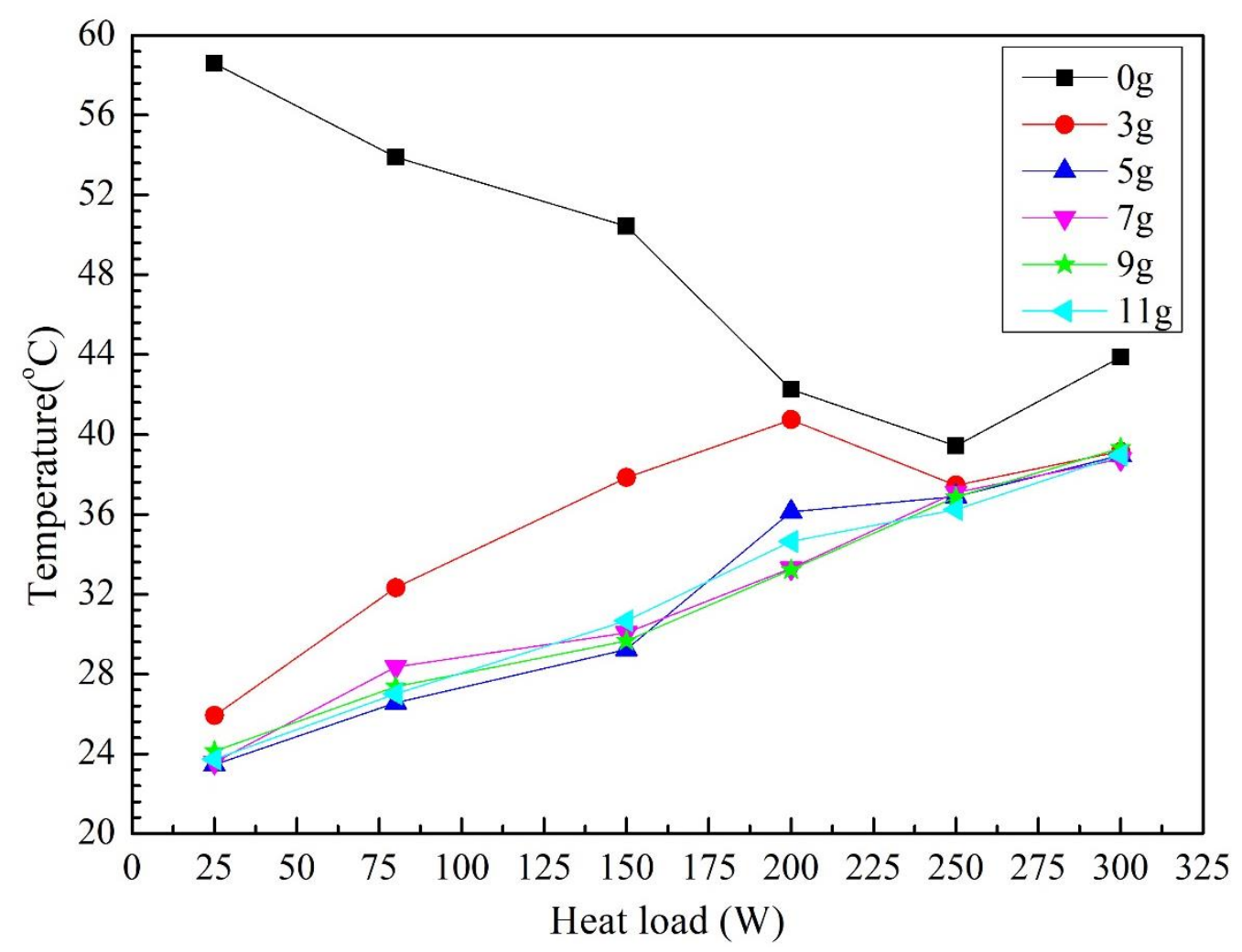

(a) Operating temperature

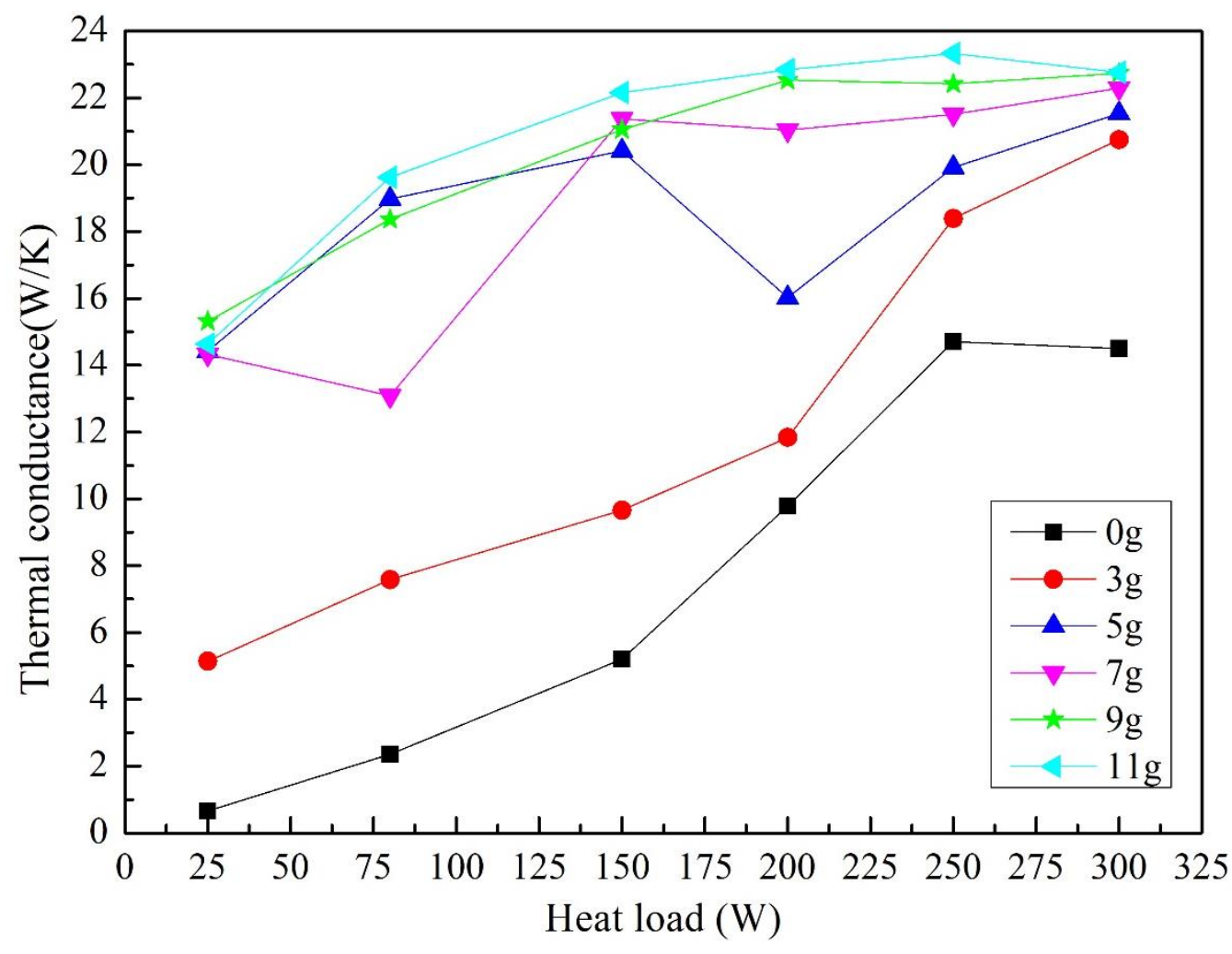

(b) Thermal conductance

Fig. 10. Steady state operating temperature and thermal conductance at loading mode II. (a)

Operating temperature. (b) Thermal conductance. 
load of $80 \mathrm{~W}$ is the smallest for all heat loads. For the case of $25 \mathrm{~W}, 80 \mathrm{~W}$ and $150 \mathrm{~W}$, the steadystate operating temperature of the DCCLHP is $23.6{ }^{\circ} \mathrm{C}, 28.4{ }^{\circ} \mathrm{C}$ and $30.1{ }^{\circ} \mathrm{C}$ respectively. The corresponding average temperature of the cold plate was $21.8^{\circ} \mathrm{C}, 22.3^{\circ} \mathrm{C}$ and $23.0^{\circ} \mathrm{C}$, respectively. Consequently, the thermal conductance was $14.3 \mathrm{~W} / \mathrm{K}, 13.1 \mathrm{~W} / \mathrm{K}$ and $21.4 \mathrm{~W} / \mathrm{K}$ at $25 \mathrm{~W}, 80 \mathrm{~W}$ and $150 \mathrm{~W}$. It could be the reason that $7 \mathrm{~g}$ acceleration leads to the higher operating temperature at $80 \mathrm{~W}$ and further leads to the smallest value of the thermal conductance. In the range from 150 $\mathrm{W}$ to $300 \mathrm{~W}$, it changes slightly. Moreover, the thermal conductance under $9 \mathrm{~g}$ and $11 \mathrm{~g}$ is similar with this change trend. At $9 \mathrm{~g}$, the thermal conductance ranges from $21.1 \mathrm{~W} / \mathrm{K}$ to $22.7 \mathrm{~W} / \mathrm{K}$ at the heat load from $150 \mathrm{~W}$ to $300 \mathrm{~W}$. At $11 \mathrm{~g}$, it ranges from $22.2 \mathrm{~W} / \mathrm{K}$ to $23.3 \mathrm{~W} / \mathrm{K}$ with the same range of the heat load. From the viewpoint of increasing the thermal conductance of the DCCLHP, the acceleration effect is desirable and advantageous to realize a low operating temperature.

\subsection{Analysis of temperature control performance}

As described previously, the temperature control performance of the DCCLHP under the above acceleration conditions shows significant difference from that under terrestrial gravity. In this section, the operating physical mechanism will be discussed in detail.

\subsubsection{Operating principle under acceleration conditions}

Based on the principle of LHP operation in gravity $[2,16,23]$, the total pressure drop of the loop includes the viscous pressure drops in vapor groove, vapor line, condenser, liquid line and wick. It is recognized that if the entire loop is not in a horizontal plane, an additional pressure head applies due to gravity and it could increase or decrease the total pressure drop. In order to drive the flow circulation, the following inequality must be satisfied.

$$
\Delta P_{\text {cap }, \text { max }} \geq \Delta P_{\text {total }}=\Delta P_{\text {vg }}+\Delta P_{\text {vl }}+\Delta P_{\text {cond }}+\Delta P_{11}+\Delta P_{\text {bay }}+\Delta P_{\text {w }}+\Delta P_{\text {a }}
$$

where $\Delta P_{\text {total }}$ is the total pressure drop in the loop, $\Delta P_{\mathrm{vg}}$ is the pressure drop in the vapor grooves, $\Delta P_{\mathrm{vl}}$ is the pressure drop in the vapor line, $\Delta P_{\text {cond }}$ is the pressure drop in the condenser, $\Delta P_{\text {IIl }}$ is the pressure drop in the liquid line, $\Delta P_{\text {bay }}$ is the pressure drop in the bayonet tube, $\Delta P_{\mathrm{w}}$ is the pressure drop in the wick, $\Delta P_{\mathrm{g}}$ is the possible additional pressure head from the gravity.

The maximum capillary pressure, which is generated from the meniscus located at the vaporliquid interface in the wick, is given by the Young-Laplace equation.

$$
\Delta P_{\text {cap } \max }=\frac{2 \sigma \cos \theta}{R}
$$


where $\sigma$ is the surface tension of the working fluid, $\theta$ is contact angle between the liquid and the wick, $R$ is the pore radius of the wick.

If the total pressure drop changes, for instance, the elevation of the evaporator with respect to the condenser varies, the capillary pressure would be self-adjusted to balance the total pressure drop by adjusting the contact angle, as shown in ineq. (2). This has been presented for adverse and positive elevation by numerous experimental and numerical studies. The corresponding operation of the LHP is named of anti-gravity operation and gravity-assisted operation $[23,25$ 28].

Similar to the gravitational pressure head, when the LHP operates under an acceleration field, an additional pressure head is also produced due to the effect of the acceleration. It will also increase or decrease the total pressure drop of the loop. Thus, the following expression needs to be satisfied to sustain the flow circulation in the loop.

$$
\Delta P_{\text {cap } \text {, max }} \geq \Delta P_{\text {total }}=\Delta P_{\text {vg }}+\Delta P_{\mathrm{vl}}+\Delta P_{\text {cond }}+\Delta P_{11}+\Delta P_{\text {bay }}+\Delta P_{\text {wick }}+\Delta P_{\text {a }}
$$

where $\Delta P_{\mathrm{a}}$ is the possible pressure head from overload acceleration. Noting that the overload acceleration may exceed the gravity acceleration for many times, the effect of the overload acceleration plays an significant role.

In the current work, the entire DCCLHP is placed on a horizontal plane, thus, the gravity effect on the operating performance can be negligible and the gravitational pressure head equals zero. For a given acceleration direction, as shown in Fig. 11, the pressure head resulted from the centrifugal acceleration will act on the working fluid in all the loop. Since the acceleration along radial direction varies, the pressure head resulted from the centrifugal acceleration is different at different position. For the purpose of simplify, an average acceleration is assumed for all the loop. As the flow direction of the working fluid is parallel to the acceleration direction, the produced pressure head will impede or promote the working fluid as an additional force. When the flow direction is perpendicular to the acceleration direction, the effect of acceleration force on the flow can be neglected. Consequently, the net pressure head gained from the acceleration can be written as:

$$
\Delta P_{\mathrm{a}}=\left(\rho_{1}-\rho_{\mathrm{v}}\right) a \Delta H
$$

where $\rho_{1}$ is the density of the liquid phase, $a$ is the radial acceleration, $\Delta H$ is the distance between 
the CCs and the vapor-liquid interface in the condenser. $\rho_{\mathrm{v}}$ is the density of the vapor phase in the vapor line and condenser as the vapor line is filled with vapor. If the vapor line is filled with vapor-liquid two-phase flow, an average density of the fluid should be used.

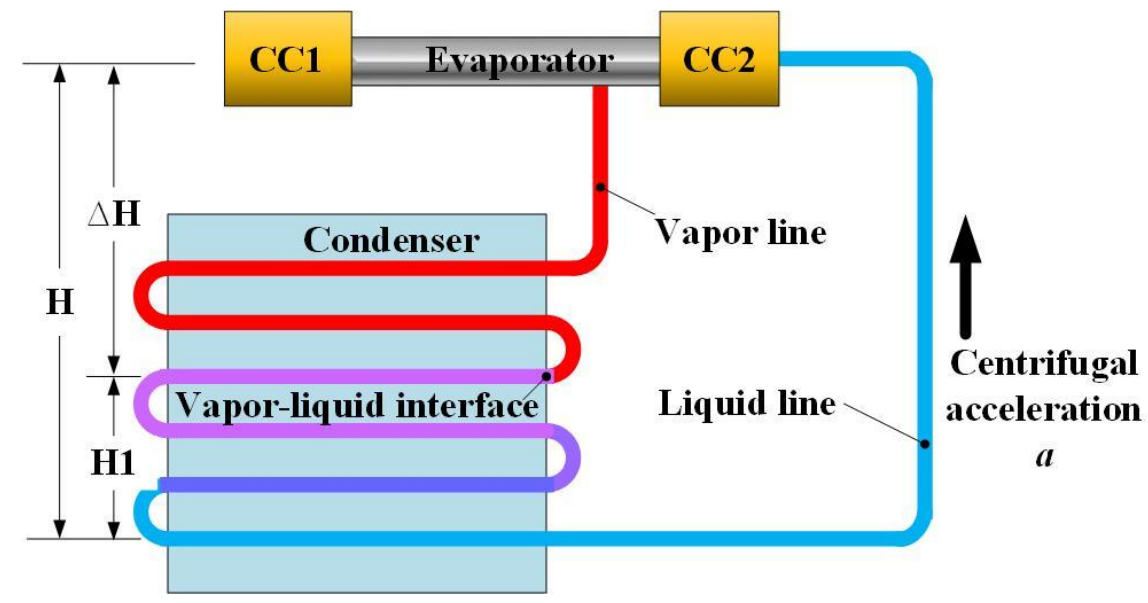

Fig. 11. Centrifugal pressure head in the loop at horizontal configuration.

Under the configuration in Fig. 11, the net pressure head becomes the driving force of the flow rather than the flow resistance. Consequently, the combined centrifugal pressure head and capillary pressure will balance the total pressure drop. The pressure balance equation of the loop can be expressed as:

$$
\Delta P_{\text {cap }}++\Delta P_{\mathrm{a}}=\Delta P_{\mathrm{vg}}+\Delta P_{\mathrm{vl}}+\Delta P_{\text {cond }}+\Delta P_{11}+\Delta P_{\text {bay }}+\Delta P_{\mathrm{w}}
$$

According to Eq. (5), for a given acceleration and heat load, the centrifugal pressure head keeps constant. As the heat load increases, so will the mass flow rate. If the total pressure drop of the loop exceeds the centrifugal pressure head, the capillary pressure is required to drive the flow circulation. The LHP operation is defined as capillary-centrifugal force co-driven mode. When the heat load decreases to a certain critical value at which the total pressure drop is equal to the centrifugal pressure head, the capillary pressure disappears with $90^{\circ}$ contact angle and horizontal meniscus. As the heat load further decreases, the centrifugal pressure head exceeds the total pressure drop and the surplus pushes forward a portion of liquid into the vapor line through the wick. As a consequence, the vapor line is filled with two-phase flow. This operation is called centrifugal force driven mode.

Under the centrifugal force driven mode, the operating characteristics of the DCCLHP are significantly different from those under the capillary-centrifugal force co-driven mode. The mass flow rate in the loop is composed of the liquid and the vapor mass flow rate. Under steady state 
conditions, the vapor mass flow rate is proportional to the heat load that applied to the evaporator. The amount of the liquid driven by the surplus of the centrifugal force automatically changes to match the pressure balance equation presented in Eq. (5). When the heat load increases, the liquid mass flow rate decreases. The actual mass flow rate of the loop is as follows:

$$
m_{\text {total }}=m_{1}+\frac{Q_{\mathrm{e}}-Q_{\mathrm{hl}}}{h}
$$

where $m_{\text {total }}$ is the total mass flow rate of the working fluid, $m_{1}$ is the mass flow rate of liquid, $Q_{\mathrm{e}}$ is the heat load applied on the evaporator, $Q_{\mathrm{hl}}$ is the heat leak from the evaporator to the CC. $h$ is the latent heat of evaporation of the working fluid.

Additionally, for the LHP operation in acceleration field, an additional inertial force will change not only the pressure balance of the loop, but also the vapor-liquid distribution, two-phase flow and heat transfer performance in the entire loop. The combination of the above impacts leads to unique operation performance of the LHP.

\subsubsection{Operation at centrifugal force driven mode}

When the LHP operates at centrifugal force driven mode, the centrifugal force becomes the sole driving force to maintain the flow circulation, which leads to the unique phenomenon as mentioned above.

For the loading mode I, the loop first operates to a steady state under terrestrial gravity before loading acceleration. The capillary force balances the total pressure drop. After the acceleration is applied, the acceleration effect can change the vapor-liquid distribution and two-phase flow in the loop and further impact on the LHP operation. The stress change of the fluid in the loop is the essence of the operation change. The centrifugal pressure head from the acceleration decreases the total pressure drop. Consequently, the capillary pressure decreases. According to Eq. (1), the evaporator temperature also decreases compared with that in terrestrial gravity, as shown in Fig. 8(a). When the acceleration is $5 \mathrm{~g}$, the centrifugal pressure head from the acceleration is large enough to balance the total viscous pressure drop at the heat load of $150 \mathrm{~W}$. In addition, the capillary pressure decreases to zero and the centrifugal force becomes the sole driving force in terms of Eq. (5). Furthermore, the centrifugal pressure head makes the absolute pressure of the liquid in the wick higher than that of the vapor in the vapor grooves. Thus, a part of liquid will be injected into the vapor grooves and downward toward the vapor line. Vapor-liquid two-phase 
flow appears in vapor grooves and vapor line besides the CCs and condenser. As a consequence, the temperatures of the vapor line, condenser and CCs are almost the same with a slight change from 29.3 to $30.4{ }^{\circ} \mathrm{C}$. While the heat load increases to $200 \mathrm{~W}$, the temperature distribution of the loop indicates that there is no liquid in the vapor line and the capillary pressure is beyond zero. The DCCLHP operates at the capillary-centrifugal force co-driven mode. Therefore, the critical heat load corresponding to the transformation from centrifugal force driven mode to capillarycentrifugal force co-driven mode is a certain value between $150 \mathrm{~W}$ and $200 \mathrm{~W}$ under $5 \mathrm{~g}$. As the acceleration increases to $7 \mathrm{~g}$, it is at $150 \mathrm{~W}$ and $200 \mathrm{~W}$ that the temperatures of the vapor line, condenser, CCs and liquid line are almost the same. It means that the DCCLHP operates at centrifugal force driven mode. When the heat load exceeds $250 \mathrm{~W}$, it operates at capillary force driven mode. Thus, the critical heat load ranges between $200 \mathrm{~W}$ and $250 \mathrm{~W}$. Based on the above analysis, it can be found that there is a critical heat load for a given acceleration, and the critical heat load increases with the acceleration increasing. Similarly, there is a critical acceleration transforming the loop operating mode for a given heat load.

For the loading mode II, the initial vapor-liquid distribution in the loop will be changed by the acceleration effect, which is different from that at loading mode I and terrestrial gravity. Therefore, the operating performance also shows an obvious difference with that at loading mode I and terrestrial gravity, which can be seen from Fig. 7 to Fig. 10 .

When the acceleration is $5 \mathrm{~g}$, the temperature of the vapor line, condenser, CCs and liquid line is within $1.0^{\circ} \mathrm{C}$ for the case of $25 \mathrm{~W}$ and $80 \mathrm{~W}$, as shown in Fig. 9(b). It indicates that there is no pure vapor in the vapor line and the DCCLHP operates at centrifugal force driven mode. However, as the heat load is $150 \mathrm{~W}$, the DCCLHP operates at capillary force driven mode. The transition heat load falls somewhere between $80 \mathrm{~W}$ and $150 \mathrm{~W}$. Increasing the acceleration to $7 \mathrm{~g}$, the DCCLHP operates at centrifugal force driven mode for the case of $25 \mathrm{~W}$ and $150 \mathrm{~W}$. But it operates at capillary force driven mode at $80 \mathrm{~W}$. This similar situation occurs at $9 \mathrm{~g}$ although the loop operates at centrifugal force driven mode at $25 \mathrm{~W}, 150 \mathrm{~W}$ and $200 \mathrm{~W}$, as shown in Fig. 9(d). As the acceleration is $11 \mathrm{~g}$, as illustrated in Fig. 9(e), the DCCLHP operates at centrifugal force driven mode for the heat load varies from $25 \mathrm{~W}$ to $200 \mathrm{~W}$. As a result, the transition heat load generally increases with the increase of the acceleration. Moreover, there exists a transition acceleration for a given heat load. 

temperature is not significant along with the acceleration under the centrifugal force driven mode. For instance, the steady-state operating temperature at $25 \mathrm{~W}$ ranges from 23.5 to $24.1{ }^{\circ} \mathrm{C}$ as the acceleration changes from $5 \mathrm{~g}$ to $11 \mathrm{~g}$. This trend can be explained as follows. Due to a two-phase flow in the vapor line, the average density of the mixture increases with the liquid mass flow rate increasing as well as increases with the acceleration increasing. Since the pressure drop in each element of the loop is directly related to the total mass flow rate, the liquid mass flow rate entering the vapor line will naturally self-adjust to satisfy Eqs. (4) and (5). Additionally, the height difference between the condenser and evaporator along the acceleration direction keeps constant. According to Eq. (5), the pressure head from the acceleration remains almost the same as the acceleration increases. For a given heat load and sink temperature, the operating temperature will also keep almost the same. The above trend is similar with the situation in terrestrial gravity reported in reference [16, 22, 23].

\subsubsection{Operation at capillary-centrifugal force co-driven mode}

When the heat load on the evaporator is larger than transition heat load, the total pressure drop in the loop is balanced by the capillary pressure. The operating principle of the DCCLHP is similar with that under the gravity-neutral configuration in terrestrial gravity. The centrifugal pressure head from the acceleration would help the flow circulation as an additional driving force. Thus, the heat transport capability of the DCCLHP will increase. Under this conditions, pure vapor occupies the vapor grooves and vapor line. It is due to the centrifugal force effect that the operation of the DCCLHP shows some differences from that under gravity.

For the case of loading mode I, when the acceleration is $1 \mathrm{~g}$ and $3 \mathrm{~g}$, the DCCLHP at different heat loads operates at capillary force driven mode. The operating temperature at $150 \mathrm{~W}$ and 200 $\mathrm{W}$ shows remarkable difference comparing the cases of terrestrial gravity, $1 \mathrm{~g}$ and $3 \mathrm{~g}$. While it shows very small differences at $250 \mathrm{~W}$ and $300 \mathrm{~W}$, even relative to the same heat load at $5 \mathrm{~g}$ and 7g. Since the entire loop researches to a steady state before applying the acceleration, the centrifugal pressure head from the acceleration can change both the pressure balance and energy balance of the loop and could make the loop reach a new equilibrium. On one hand, the centrifugal pressure head increases the absolute pressure in the CCs and decreases the pressure 
elements decreases according to Eq. (1), and the heat leak from the evaporator to CCs reduces. On the other hand, the liquid quantity in the CCs at small heat load is much less than that at large heat load. This vapor-liquid distribution is easily affected by the acceleration effect and might cause the heat leak decrease. Moreover, the liquid temperature at the exit of the liquid line remains almost the same and the subcooling carried by the returning liquid remains unchanged. Therefore, the evaporator temperature will show obvious drop at $150 \mathrm{~W}$ and $200 \mathrm{~W}$. For the cases of $250 \mathrm{~W}$ and $300 \mathrm{~W}$, the capillary force is much larger. The change of the pressure difference between the evaporator and CCs and the heat leak is not significant caused by the centrifugal force. Thus, the evaporator temperature shows slightly change.

For the case of loading mode II, when the acceleration is $3 \mathrm{~g}$, the DCCLHP operates at capillary force driven mode at different heat loads. Comparing to the case in gravity, the evaporator core could be filled with liquid. The centrifugal pressure head would lead to the absolute pressure increase in the CCs. This in turn leads to the pressure difference between the evaporator and CCs to raise. Consequently, a smaller temperature difference between both two elements is occurred, which further results in a lower heat leak from the evaporator to CCs. Moreover, the mass flow rate and the liquid temperature at the exit of the liquid line keep almost constant. The subcooling returning to the CCs also keeps almost constant. As a result, the evaporator temperature drops. Comparing the cases at different accelerations, it can be found that the condenser is not fully used as the loop operates at capillary force driven mode. It can be seen that for the cases of $250 \mathrm{~W}$ and $300 \mathrm{~W}$, the vapor-liquid interface is close to the end of the condenser. Hence, the change of height difference $\Delta H$ is small and can be neglected. When the acceleration increases, the temperature difference between the evaporator and CCs decreases and the heat leak from the evaporator to CCs decreases. But the subcooling of returning liquid also reduces due to the liquid temperature at the exit of the liquid line raises. Therefore, the operating temperature maybe change slightly.

Here it is worth noting that the operating temperature of the DCCLHP at $250 \mathrm{~W}$ or $300 \mathrm{~W}$ remains almost the same under different acceleration conditions regardless of either loading mode. From the viewpoint of controlling the temperature of the airborne electronic devices, the capillary force driven mode is preferable as at this mode the almost constant operating temperature is obtained. 


\section{Conclusions}

The operating characteristics of the DCCLHP under acceleration force-assisted conditions were investigated both experimentally and theoretically. The various impact factors such as heat load, acceleration magnitude and loading mode were analyzed in a systematic manner. The operating principle under acceleration conditions was developed. Analyses of the theoretical and experimental results lead to the following main conclusions:

(1) The effect of the assisted acceleration force can improve the operation of the DCCLHP compared to that under terrestrial gravity field. The operating temperature will be lower at a small heat load when the acceleration is higher. But at a large heat load, the change of the operating temperature is insignificant with the acceleration.

(2) The classic "V-shape" curve of the operating temperature with heat load gradually degenerates to "/-shape" oblique line with the acceleration increase for both loading modes. The operating temperature at loading mode II is less than that at loading mode I. It is expected that for large acceleration and small heat load, the temperature difference of all the loop become extremely small at the loading mode II.

(3) The DCCLHP under acceleration force assisted condition can operate at centrifugal force driven mode or capillary-centrifugal force co-driven mode. The transition heat load from at centrifugal force driven mode to at capillary-centrifugal force co-driven mode increases with the acceleration increase.

(4) The unique operating characteristics of the DCCLHP under acceleration force assisted condition are the outcome of the combined action of the loop pressure rebalance, vapor-liquid distribution, two-phase flow and heat transfer caused by acceleration effect.

\section{Acknowledgement}

The authors would like to acknowledge the financial support from the Fundamental Research Funds for the Central Universities of China (YWF-14-HKXY-019). We also acknowledge the financial support from the UK Fluids Network. 


\section{References}

765

766

767

[1] Maydanik Y F. Loop heat pipes. Applied Thermal Engineering, 2005, 25(5): 635-657.

[2] Ambirajan A, Adoni A A, Vaidya J S, Rajendran A A, Kumar D, Dutta P. Loop heat pipes: a review of fundamentals, operation, and design. Heat Transfer Engineering, 2012, 33(4-5): $387-405$.

[3] Siedel B, Sartre V, Lefèvre F. Literature review: Steady-state modelling of loop heat pipes. Applied Thermal Engineering, 2015, 75: 709-723.

[4] Chernysheva M A, Maydanik Y F. Simulation of heat and mass transfer in a cylindrical evaporator of a loop heat pipe. International Journal of Heat and Mass Transfer, 2019, 131: 442-449.

[5] Wang H, Lin G, Shen X, Bai L, Wen D. Effect of evaporator tilt on a loop heat pipe with noncondensable gas. International Journal of Heat and Mass Transfer, 2019, 128: 1072-1080.

[6] Zhang H, Li G, Chen L, Man G, Miao J, Ren X, He J, Huo Y. Development of Flat-Plate Loop Heat Pipes for Spacecraft Thermal Control. Microgravity Science and Technology, 2019, 31(4): 435-443.

[7] Y.F.Maydanik, S.V.Vershinin, M.A.Chernysheva. Investigation of thermal characteristics of a loop heat pipe in a wide range of external conditions. International Journal of Heat and Mass Transfer, 2020 (147): 118967.

[8] Zhang Q, Lin G, Shen X, Wen D. Visualization study on the heat and mass transfer in the evaporator-compensation chamber of a loop heat pipe. Applied Thermal Engineering, 2020, 164: 114472

[9] Su Q, Chang S, Song M, Zhao Y, Dang C. An experimental study on the heat transfer performance of a loop heat pipe system with ethanol-water mixture as working fluid for aircraft anti-icing. International Journal of Heat and Mass Transfer, 2019, 139: 280-292.

[10] Ku J, Nagano H. Effects of gravity on start-up and heat load sharing of a miniature loop heat pipe. SAE Technical Paper, No.2007-01-3234, 2007.

[11]Zhou G, Li J. Two-phase flow characteristics of a high performance loop heat pipe with flat evaporator under gravity. International Journal of Heat and Mass Transfer, 2018, 117: 1063 1074. 
[12] Ku J. Pressure profiles in a loop heat pipe under gravity influence. 45th International Conference on Environmental Systems, No. ICES-2015-002, 2015.

[13] Watanabe N, Phan N, Saito Y, Hayashi S, Katayama N, Nagano H. Operating characteristics of an anti-gravity loop heat pipe with a flat evaporator that has the capability of a loop thermosyphon. Energy Conversion and Management, 2020, 205: 112431.

[14]Chen Y, Groll M, Mertz R, Maydanik Y F, Vershinin S V. Steady-state and transient performance of a miniature loop heat pipe. ASME 3rd International Conference on Microchannels and Minichannels, American Society of Mechanical Engineers, 2005: 183189.

[15] Ku J. Operating characteristics of loop heat pipes. SAE Technical Paper No. 1999-01-2007, 1999.

[16] Chuang P Y A, Cimbala J M, Brenizer J S. Experimental and analytical study of a loop heat pipe at a positive elevation using neutron radiography. International Journal of Thermal Sciences, 2014, 77: 84-95.

[17]Chuang P Y A. An improved steady-state model of loop heat pipes based on experimental and theoretical analyses. PhD thesis, The Pennsylvania State University, 2003.

[18]Riehl R R. Comparing the behavior of a loop heat pipe with different elevations of the capillary evaporator. SAE Technical Paper No. 2004-01-2510, 2004.

[19]Chang X, Watanabe N, Nagano H. Visualization study of a loop heat pipe with two evaporators and one condenser under gravity-assisted condition. International Journal of Heat and Mass Transfer, 2019, 135: 378-391.

[20]Chuang P Y A, Cimbala J M, Brenizer Jr J S, Conroy C T, El-Ganayni, A A, Riley, D R. Comparison of experiments and 1-D steady-state model of a loop heat pipe. Proceedings of IMECE, New Orleans, LA USA, 2002: 17-22.

[21]Mo D C, Ding N, Lu S S. Gravity effects on the performance of a flat loop heat pipe. Microgravity Science and Technology, 2009, 21: 95-102.

[22] Bai L Z, Lin G P, Zhang H X. Experimental study on steady state operating characteristics of gravity assisted loop heat pipes. Acta Aeronautica et Astronautica Sinica, 2008, 29: 11121117 (In Chinese).

[23]Bai L, Guo J, Lin G, He J, Wen D. Steady-state modeling and analysis of a loop heat pipe 
under gravity-assisted operation. Applied Thermal Engineering, 2015, 83: 88-97.

[24]Bai L, Tao Y, Guo Y, Lin G. Startup characteristics of a dual compensation chamber loop heat pipe with an extended bayonet tube. International Journal of Heat and Mass Transfer, 2020, 148: 119066.

[25]Feng J T, Lin G P, Bai L Z. Experimental investigation on operating instability of a dual compensation chamber loop heat pipe. Science in China Series E: Technological Sciences, 2009, 52(8): 2316-2322.

[26] Ku J, Garrison M, Patel D, Robinson F, Ottenstein L. Loop heat pipe temperature oscillation induced by gravity assist and reservoir heating. 45th International Conference on Environmental Systems, No. ICES-2015-003, 2015.

[27] Ku J, Ottenstein L, Kaya T, Rogers P, Hoff C. Testing of a loop heat pipe subjected to variable accelerating forces, Part 1: Start-up. SAE Technical Paper, No. 2000-01-2488, 2000.

[28] Ku J, Ottenstein L, Kaya T, Rogers P, Hoff C. Testing of a loop heat pipe subjected to variable accelerating forces, Part 2: Temperature stability. SAE Technical Paper, No. 2000-01-2489, 2000.

[29]Fleming A J, Thomas S K, Yerkes K L. Titanium-water loop heat pipe operating characteristics under standard and elevated acceleration fields. Journal of Thermophysics and Heat Transfer, 2010, 24(1): 184.

[30] Yerkes K L, Scofield J D, Courson D L, Jiang H. Steady-periodic acceleration effects on the performance of a loop heat pipe. Journal of Thermophysics and Heat Transfer, 2014, 28(3): 440-454.

[31] Yerkes K L, Scofield J D, Courson D L. Performance of a loop heat pipe subjected to a phasecoupled heat input to an acceleration field. The 46th AIAA Thermophysics Conference. 2016: 4145.

[32] Xie Y Q, Zhang J, Xie L Y, Yu Y, Wu H W, Zhang H X, Gao H X. Experimental investigation on the operating characteristics of a dual compensation chamber loop heat pipe subjected to acceleration field. Applied Thermal Engineering, 2015, 81: 297-312.

[33]Xie Y Q, Zhou Y, Wen D S, Wu H W, Haritos G, Zhang H X. Experimental investigation on transient characteristics of a dual compensation chamber loop heat pipe subjected to acceleration forces. Applied Thermal Engineering, 2017, 130: 169-184. 